\title{
Accretion Processes in Astrophysics: The State of Art
}

\author{
Franco Giovannelli ${ }^{* \dagger}$ \\ INAF - Istituto di Astrofisica e Planetologia Spaziali, Via del Fosso del Cavaliere, 100, 00133 \\ Roma, Italy \\ E-mail: Eranco.giovannellidiaps.inaf.it \\ Lola Sabau-Graziati \\ INTA-Dpt. Cargas Utiles y Ciencias del Espacio, C/ra de Ajalvir, Km 4 - E28850 Torrejón de \\ Ardoz, Madrid, Spain \\ E-mail: sabaumdeinta.es
}

In this review paper we will discuss about the accretion processes that regulates the growth and evolution of all objects in the Universe. We will make a short cruise discussing the accretion in Young Stellar Objects (YSOs), in Planets (Pts), in White Dwarfs (WDs), in Neutron Stars (NSs), and in Black Holes (BHs) independent of their masses. In this way we will mark the borders of the arguments discussed during this workshop on Accretion Processes in Cosmic Sources: Young Stellar Objects, Cataclysmic Variables and Related Objects, X-ray Binary Systems, Active Galactic Nuclei.

This paper gets information from updated versions of the book "The Impact of the Space Experiments on Our Knowledge of the Physics of the Universe" published in 2004 by the Kluwer Academic Publishers, reprinted from the review paper by Giovannelli, F. \& Sabau-Graziati, L.: 2004, Space Sci. Rev. 112, 1-443 (GSG2004), and subsequent considered lucubrations.

The review is a source of a huge amount of references. Thus, who wants to enter in details in one of the discussed arguments can find an almost exhaustive list of specific references.

Accretion Processes in Cosmic Sources "APCS2016"

5-10 September 2016,

Saint Petersburg, Russia

*Speaker.

${ }^{\dagger}$ A footnote may follow. 


\section{Introduction}

Accretion disks - mainly protoplanetary disks and those of binary stars - are of extreme interest, since they demonstrate the great variety of observational manifestations and allow studying a big number of physical processes. The main question about the mechanism of the angular momentum transfer in the disks has not been completely solved yet, though the disks have been attracting the interest of many scientists for several decades. From observations, one can derive the rate of accretion onto the central object, which enables determining the rate of angular momentum loss in the disk. If one supposes that the angular momentum transfer is driven solely by the material viscosity in the disk, then one finds that the viscosity coefficient in the disks should be anomalously high, by 9-11 orders of magnitude higher than the coefficient of molecular viscosity. To answer the question, whether the angular momentum transfer is driven by developed turbulence in the disk, and, if so, to understand the nature of the turbulent viscosity, one should be able, if possible, to derive the parameters of turbulence from observational data (Bisikalo et al., 2016 and the references therein).

\subsection{Some rightful historical remarks}

When the first theories about accretion disks around compact stars started to be developed around the 1960s, the class of the so-called Cataclysmic Variables (CVs) started to have a leading position in astrophysics. They constituted the perfect laboratories for testing those theories. When the UV window to the universe was opened at the end of 1970s with the advent of the historical IUE (International Ultraviolet Explorer), CVs became really one of the most interesting class of objects of the whole astrophysics.

Previously, essentially two schools of thought born in Cambridge (UK) and in Warsaw (Poland) in order to tackle with the difficult subject of the mass exchange in close binary systems (e.g. Smak, 1962, 1971, 1981a,b, 1984a,b; Paczynski, 1965, 1977; Bath, 1969, 1975, 1976, 1978, 1980, 1984a,b and the references therein; Bath et al., 1974; Mantle \& Bath, 1983). However, two fundamental papers about accretion disks appeared at the beginning of 1970s (Shakura, 1972, Shakura $\&$ Sunyaev, 1973). These papers marked substantially the development of theories about accretion disks around compact objects in binary systems, until present times. Pringle (1981) reviewed accretion disks in astrophysics (see Giovannelli, 2008).

\subsection{Accretion processes}

Accretion is a universal phenomenon that takes place in the vast majority of astrophysical objects. The progress of ground-based and space-borne observational facilities has resulted in the great amount of information on various accreting astrophysical objects, collected within the last decades. The accretion is accompanied by the process of extensive energy release that takes place on the surface of an accreting object and in various gaseous envelopes, accretion disk, jets and other elements of the flow pattern. The results of observations inspired the intensive development of accretion theory, which, in turn, enabled us to study unique properties of accreting objects and physical conditions in the surrounding environment. One of the most interesting outcomes of this intensive study is the fact that accretion processes are, in a sense, self-similar on various spatial 
scales from planetary systems to galaxies. This fact gives us new opportunities to investigate objects that, by various reasons, are not available for direct study.

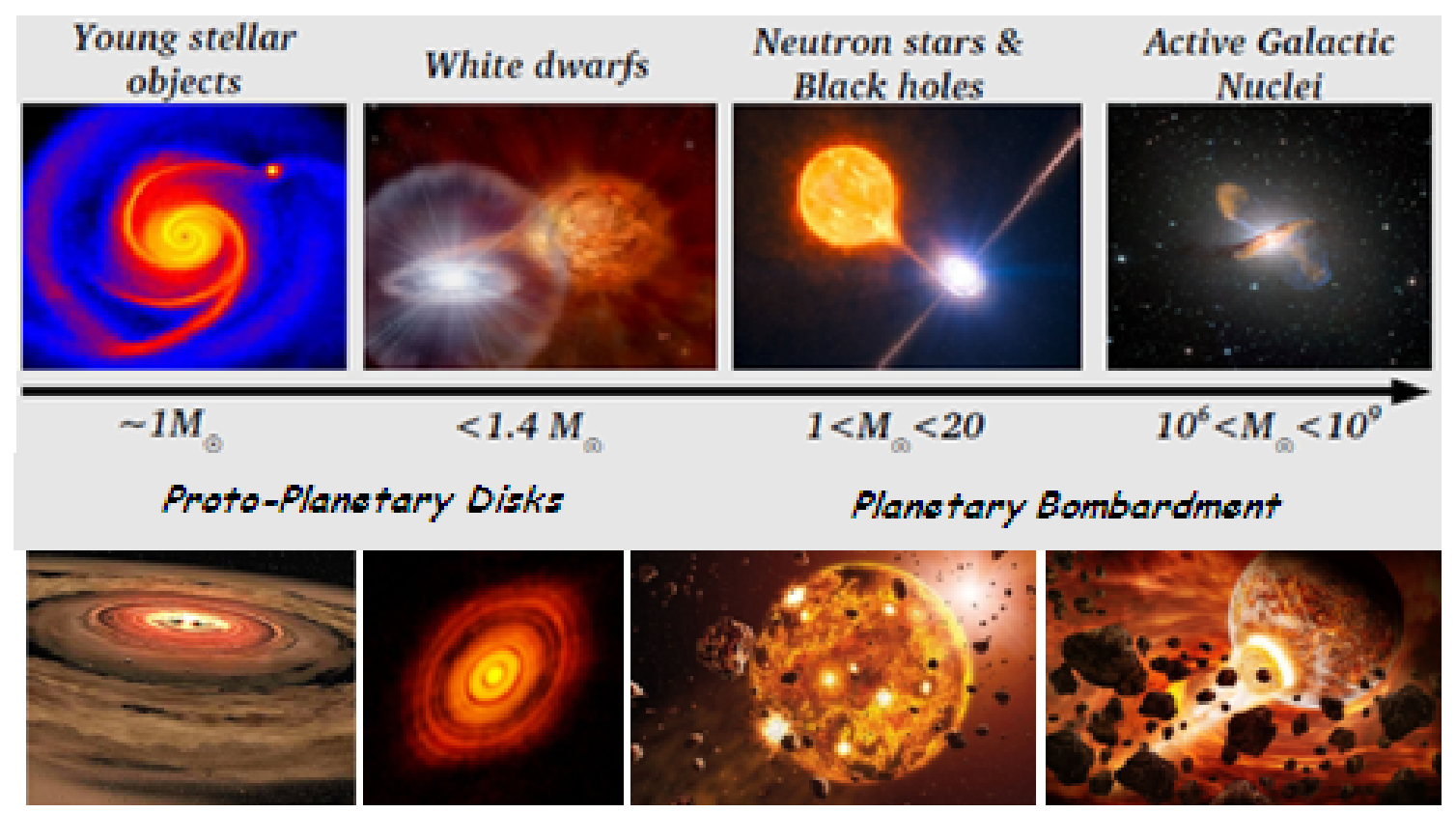

Figure 1: Accretion processes in different cosmic sources (after Scaringi, 2015).

Cataclysmic variable stars are unique natural laboratories where one can conduct the detailed observational study of accretion processes and accretion disks.

Figure 1 shows a sketch of cosmic systems where accretion processes occur (after Scaringi, 2015).

Accretion disks are the most common mode of accretion in astrophysics. The strong gravitational force of the compact object attracts matter from the companion. The infalling matter have some angular momentum and conservation of angular momentum prevents matter from falling directly into the compact object.

Accretion disks are flattened astronomical objects made of rapidly rotating gas which slowly spirals onto a central gravitating body. The gravitational energy of infalling matter extracted in accretion disks powers stellar binaries, active galactic nuclei, proto-planetary disks and some GRBs.

In accretion disks the high angular momentum, J, of rotating matter is gradually transported outwards by stresses (related to turbulence, viscosity, shear and magnetic fields). This gradual loss of $\mathrm{J}$ allows matter to progressively move inwards, towards the centre of gravity. The gravitational energy of the gaseous matter is thereby converted to heat. A fraction of the heat is converted into radiation, which partially escapes and cools down the accretion disk. Accretion disk physics is thus governed by a non-linear combination of many processes, including gravity, hydrodynamics, viscosity, radiation and magnetic fields (e.g. Menou, 2008).

The observable physical quantity of radiation produced in accretion disks is the luminosity. As photons carry momentum and thus can exert pressure there is a maximum possible luminosity at which gravity is able to balance the outward pressure of radiation. The limit for a steady, spherically symmetric accretion flow is given by the Eddington luminosity $\left(\mathrm{L}_{\mathrm{Edd}}\right)$. For a typical mass of a NS 
$\left(\mathrm{M}_{\mathrm{NS}} \simeq 1.4 \mathrm{M}_{\odot}\right), \mathrm{L}_{\mathrm{Edd}} \simeq 2 \times 10^{38} \mathrm{erg} \mathrm{s}^{-1}$ (Shapiro \& Teukolsky, 1983). Since accretion disks are not spherical and often have additional stresses that can counteract the radiation pressure along with gravity, they may be brighter than this limit and radiate at super-Eddington luminosity (e.g. Pacucci, Volonteri \& Ferrara, 2015; Pacucci, 2016; Sakurai, Inayoshi \& Haiman, 2016).

Many papers, reviews, and books have been published about the accretion processes in cosmic sources (e.g. Shakura, 1972; Shakura \& Sunyaev, 1973; Pringle, 1981; Frank, King \& Raine, 1985, 1992, 2002; Lewin, van Paradijs \& van den Heuvel, 1995; Papaloizou \& Lin, 1995; Lin \& Papaloizou, 1996; Lynden-Bell, 1996; Meyer-Hofmeister \& Spruit, 1997; Hartmann, 1998; Chakrabarti, 2004; Abramowicz \& Fragile, 2013; González Martínez-País, Shahbaz \& Casares Velázquez, 2014; Falanga et al., 2015; Lasota, 2016; Hartmann, Herczeg \& Calvet, 2016).

\section{Star Formation}

Our current understanding of the physical processes of star formation has been reviewed by Larson (2003), with emphasis on processes occurring in molecular clouds like those observed nearby. The dense cores of these clouds are predicted to undergo gravitational collapse characterized by the runaway growth of a central density peak that evolves towards a singularity. As long as collapse can occur, rotation and magnetic fields do not change this qualitative behaviour. The result is that a very small embryonic star or protostar forms and grows by accretion at a rate that is initially high but declines with time as the surrounding envelope is depleted. Rotation causes some of the remaining matter to form a disk around the protostar, but accretion from protostellar disks is not well understood and may be variable. Most, and possibly all, stars form in binary or multiple systems in which gravitational interactions can play a role in redistributing angular momentum and driving episodes of disk accretion. Variable accretion may account for some peculiarities of young stars such as flareups and jet production, and protostellar interactions in forming systems of stars will also have important implications for planet formation. The most massive stars form in the densest environments by processes that are not yet well understood but may include violent interactions and mergers. The formation of the most massive stars may have similarities to the formation and growth of massive black holes in very dense environments.

It is important to say some words about the pathway that matter covers from interstellar medium (ISM) up to form a star.

The process that governs the global structure from large-scale properties of the ISM to stars is called fragmentation. It is a hierarchical process in which parent clouds break up into subclouds, which may themselves break into smaller structures. It is a multiscale process (Efremov \& Elmegreen, 1998) that ranges from scales of:

- $10 \mathrm{kpc}$ - spiral arms of the Galaxy;

- $1 \mathrm{kpc}-\mathrm{H}$ I super clouds;

- 100 pc-giant molecular clouds;

- $10 \mathrm{pc}$ - molecular clouds;

- 0.1 pc-molecular cores; 
- $100 \mathrm{AU}$ - protostars.

Figure 2 shows a schematic diagram of the sizes and durations of star formation in different regions (Efremov \& Elmegreen, 1998).

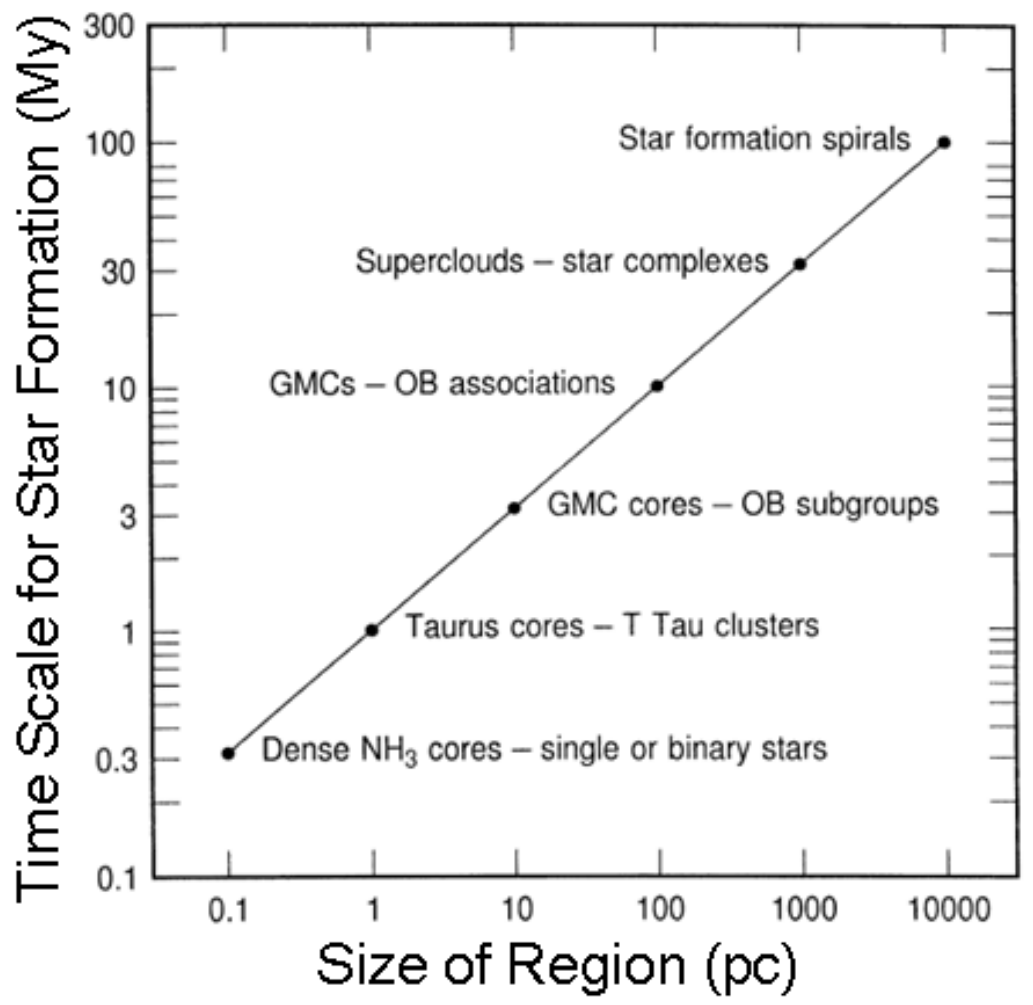

Figure 2: A schematic diagram of the sizes and durations of star formation in different regions (adapted from Efremov \& Elmegreen, 1998).

Stars are the final step of fragmentation. The first four items are large-scale structures in terms of fragmentation and their stability is determined by thermal, magnetic and turbulent support. The last two items are small-scale structures and are predominantly supported by thermal and magnetic pressure. Open issues relate to questions of whether magnetic fields can really prevent or at least delay gravitational collapse. A deep discussion can be found in the paper by Elmegreen (2002) and in the book of Schulz (2012).

Specifically, stars had to evolve as a strong function of their mass. Key were hydrogen fusion lifetimes. From early calculations it was easily recognized that low-mass stars can burn hydrogen for billions of years. On the other hand, massive stars radiate energy at a rate which is many orders of magnitude larger than low-mass stars. Since their mass is only a few ten times larger this means that their fusion lifetime is much smaller. In fact, for massive $\mathrm{O}$ stars it is of the order of ten million years and less. Stars burn about $10 \%$ of their hydrogen and as a rule of thumb one can estimate the lifetime of stars by:

$$
\mathrm{T}_{\text {life }}=7.3 \times 10^{9} \times \frac{\mathrm{M} / \mathrm{M}_{\odot}}{\mathrm{L} / \mathrm{L}_{\odot}}
$$


where: $\mathrm{M}_{\odot}$ and and $\mathrm{L} \odot$ are the mass and luminosity of the Sun and $\mathrm{M}$ and $\mathrm{L}$ the same for the star (Unsoeld \& Baschek, 2001). Clearly, this development put an end to the perception that the Draper classification could resemble an evolutionary scale where early type $\mathrm{O}$ stars evolve into late type stars.

However, it is interesting to remark the importance of magnetic fields from diffuse media to compact sources. The book Magnetic Fields in Diffuse Media (Lazarian, de Gouveia Dal Pino \& Melioli, 2015) presents the current knowledge of magnetic fields in diffuse astrophysical media. Starting with an overview of twenty-first century instrumentation to observe astrophysical magnetic fields, the chapters cover observational techniques, origin of magnetic fields, magnetic turbulence, basic processes in magnetized fluids, the role of magnetic fields for cosmic rays, in the interstellar medium and for star.

Indeed, astrophysical plasmas are magnetized and turbulent and this presents a serious challenge for studying such a media. For decades turbulent astrophysical magnetic fields were completely enigmatic and this was constraining the progress in understanding key astrophysical processes. In particular, in diffuse media, the processes of cosmic ray propagation, heat transport, formation of a variety of density structures, filaments, molecular clouds, etc. were treated using poorly constrained values of magnetic fields and somewhat ad hoc models of magnetic turbulence. The essential processes related to magnetic fields, for instance, the ability of magnetic fields to change their topology, i.e. magnetic reconnection, were hotly debated with the estimates of the rates used by different researchers varying by many orders of magnitude.

A substantial progress has been made in the field of magnetic fields in diffuse media in the last decade. The new observations allowed to map magnetic fields with higher precision, which affected substantially our understanding of the role of magnetic fields in the interstellar medium and intracluster medium. With better computational abilities it became possible to test and reject theories of key processes in magnetized media. In particular, essential progress has been achieved in understanding the origin and evolution of magnetic fields, in the theory of magnetic field generation, in the physics of magnetic reconnection, acceleration and propagation of cosmic rays in turbulent magnetized media.

\section{Accretion in Young Stellar Objects}

The study of Pre-Main Sequence Stars (PMSSs) is of great interest as it provides crucial information on stellar evolution and, particularly, on the role of magnetic fields, angular momenta, accretion of matter, and mass loss processes, as well as indirect information on the formation processes of the Sun and Solar system.

Before the space era, practically all observations of PMSSs were only restricted at the optical and near-IR (NIR) spectral regions. With the nowdays knowledge of PMSSs, we can say that they are sites of a variety of extraordinary astrophysical processes, including accretion disks and outflows. The presence of an accretion disk have been strengthened by the observation of nearly Keplerian differential rotation (Hartmann \& Kenyon, 1987a,b) in the optical and NIR spectra of FU Ori, a young stellar object (YSO) whose spectral energy distribution (SED) can be explained by the presence of a disk. 
PMS is an important evolutionary phase of all stars, which are evolving from their birth to the Main Sequence (MS), and then to their ultimate fate. A first important difference among stars is due to their masses (or luminosity). Indeed, as well known, high mass stars evolve rapidly into the MS and therefore the experimental study of their evolution within the PMS phase is very difficult, due to the lack of a good statistics. On the contrary, low mass stars, because of their slower evolution towards the MS, can be studied in details since it is possible to find large samples of objects belonging to different stages of the PMS evolutionary phase (for details see e.g. Giovannelli, 1994).

The evolution of YSOs has four different phases: Class 0, Class I, Class II and Class III. Class 0 is observable only in submm range. Class I, II and III sources are characterized with a blackbody radiation and an infrared excess. The amount of infrared excess is decreasing during the evolution. Class II and III objects are also visible in the optical bands (see Fig. 3).

Class 0 objects are sources with a central protostar that are extremely faint in the optical and

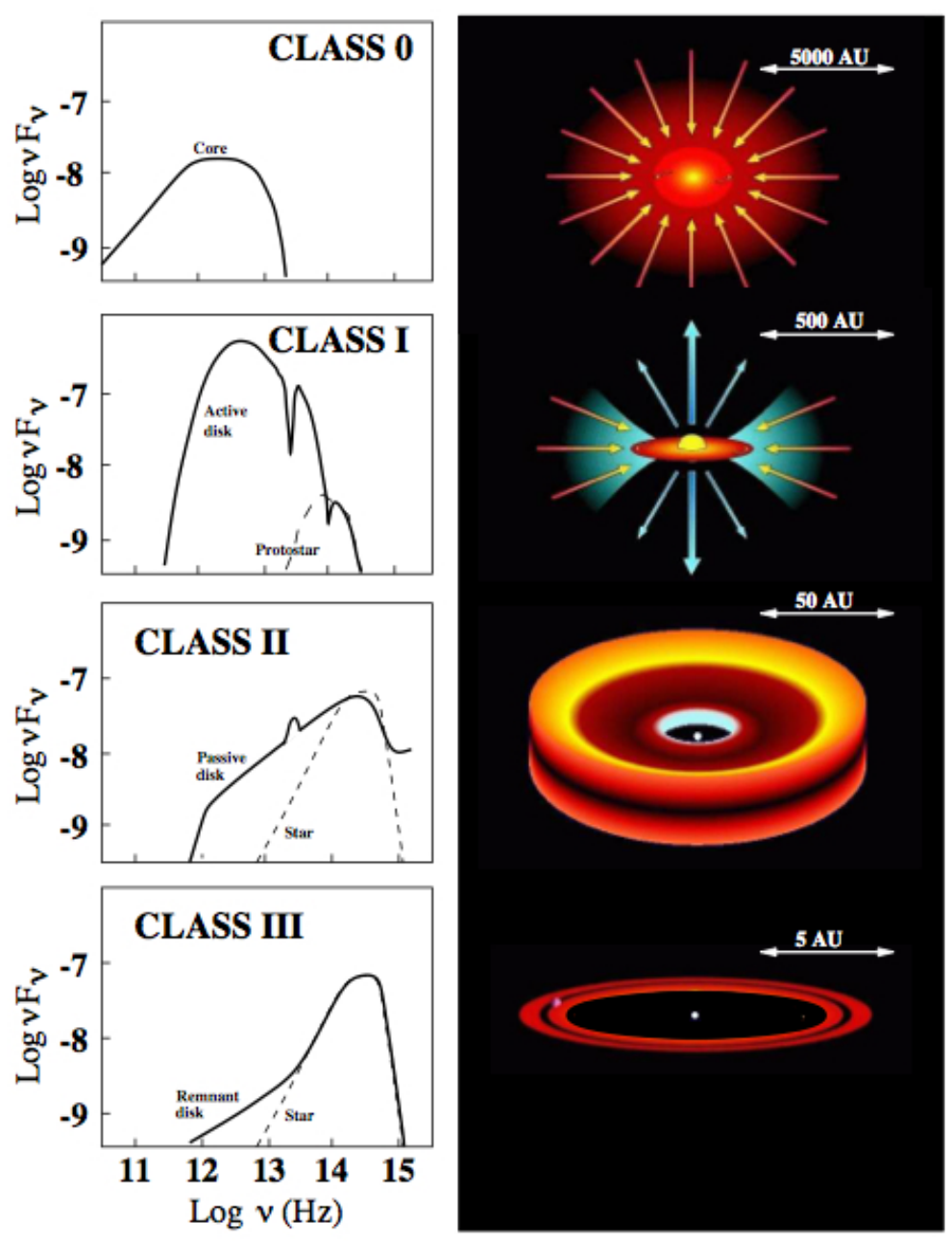

Figure 3: Sketch of the formation of a single star and the evolution of the circumstellar material. The left panels show the SED of the system at different evolutionary stages. The right panels show the corresponding system geometry (adopted from Andrea Isella, 2006, after André, 1994). 
near-IR and that have a significant submillimeter luminosity. Class I sources (a $>0$ ) are relatively evolved embedded sources with circumstellar disks and envelopes, they are optically invisible stars with SEDs that peak at mid-IR to far-IR wavelengths. Class II, or classical T Tauri stars $(-2<\mathrm{a}$ $<0)$ have significant circumstellar disks, strong emission lines and substantial IR or UV excesses characterized them. Class III, weak emission T Tauri stars $(\mathrm{a}<-2)$ have weak or no emission lines and negligible excesses, they are no longer accreting significant amounts of matter.

Stellar properties of embedded protostars have been reviewed by White et al. (2007) starting from the objective fact that protostars are precursors to the nearly fully assembled T-Tauri and Herbig Ae/Be type stars undergoing quasi-static contraction towards the zero-age main sequence; they are in the process of acquiring the majority of their stellar mass. Although numerous young stars with spatially extended envelope-like structures appear to fit this description, their high extinction has inhibited observers from directly measuring their stellar and accretion properties and confirming that they are in fact in the main phase of mass accretion (i.e., true protostars).

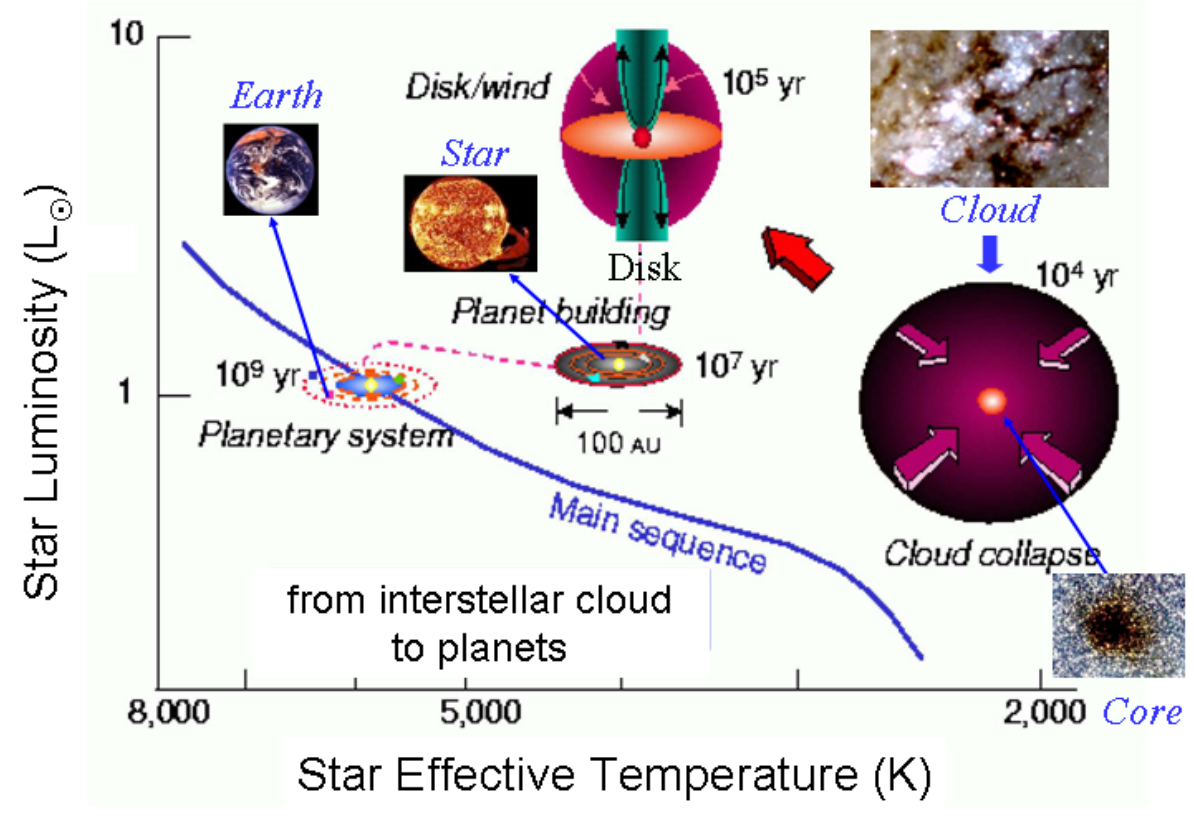

Figure 4: The main stages of planetary formation from the collapse of an interstellar cloud (adapted from Beckwith \& Sargent (1996).

Figure 4 shows even better the pathway for the formation of planets from an interstellar (IS) cloud in the case of Solar System (Beckwith \& Sargent, 1996). From the collapse of the IS cloud in a timescale of order $10^{4} \mathrm{yr}$ that forms a core, through the active disk/outflow phase $\left(\approx 10^{5} \mathrm{yr}\right)$, the passive disk/planet formation phase $\left(\approx 10^{7} \mathrm{yr}\right)$, and the existence of a stable planetary system by the time the star reaches the MS $\left(\approx 10^{9} \mathrm{yr}\right)$. The formation of the disk occurs naturally. The disk acts as a reservoir for material with too much initial angular momentum to fall onto the central star. Disks emit and reflect light very well and can be seen to relatively large distances from the central star with the new instrumentation both ground- and space-based in different energy regions.

A discussion about the structure and evolution of pre-main-sequence circumstellar disks can be found in the paper by Isella, Carpenter \& Sargent (2009), and a deep discussion about the 
formation and evolution of planetary systems can be found in the paper by Carpenter et al. (2009) in which they used Spitzer photometric and spectroscopic observations.

\subsection{Disk observations}

Circumstellar disks can be detected by several techniques, each tracing specific features, temperatures and/or regions of the disk. Combining this knowledge enables us not only to proof the existence of disks but also to probe the physics which is happening inside of them. Most of the disk mass is expected to reside in gas (because disks are made out of interstellar material), however this component is the hardest to detect as it is often frozen out, especially in the outer, cold parts of the disk and in the mid-plane. The disk geometry and the according spectral energy distribution (SED) is schematically shown in Fig. 5. In fact this IR excess was the first observational evidence for disks around young stars (e.g., Strom et al. 1989; Beckwith et al. 1990).

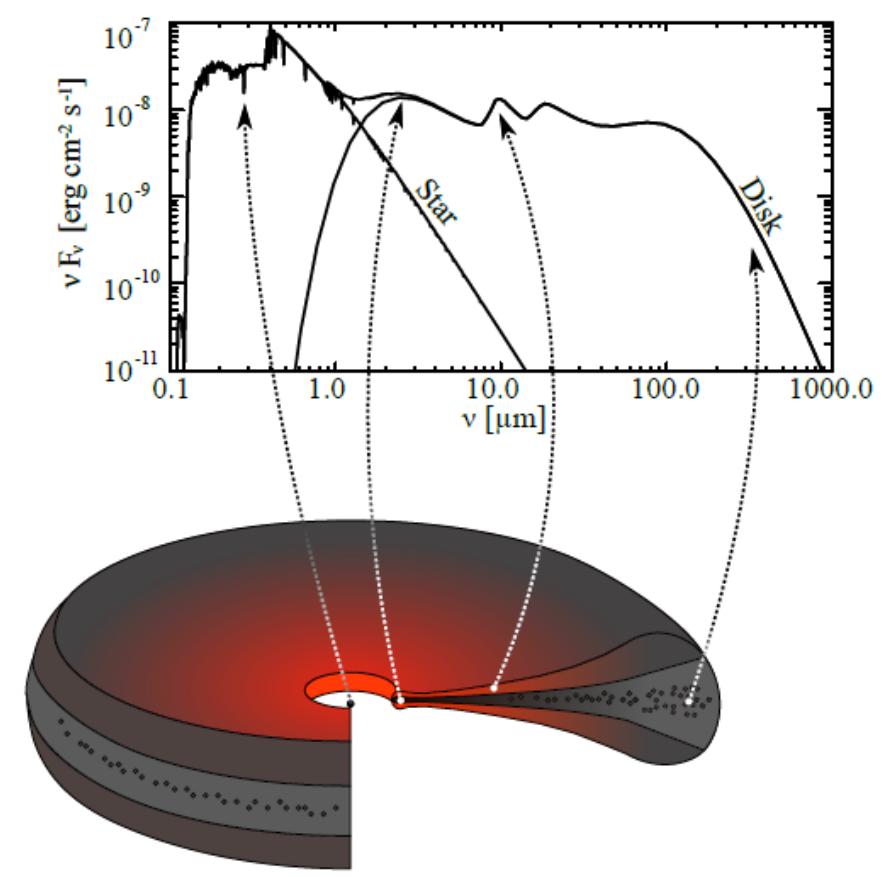

Figure 5: Origin of the spectral energy distribution: at larger wavelength, the disk emission outweighs the stellar spectrum. Generally, the shorter wavelength emission comes from hotter, inner regions of the disk. Large wavelength (submillimeter) probe the dust mass in the outer, optically thin regions (adopted from Birnstiel, 2010, after from Dullemond et al., 2007).

Wolf et al. (2012) present a review of the interplay between the evolution of circumstellar disks and the formation of planets, both from the perspective of theoretical models and dedicated observations. They discuss fundamental questions concerning the formation and evolution of circumstellar disks and planets which can be addressed in the near future with optical and IR long-baseline interferometers, as well as the importance of complementary observations with longbaseline (sub)millimiter interferometers and high-sensitivity IR observations.

How do we know that disks exist? We have many inferences and proofs, like shown in Fig. 6 (after Beckwith, 1998). 


\section{How do we know disks exist?}

\section{Inference}

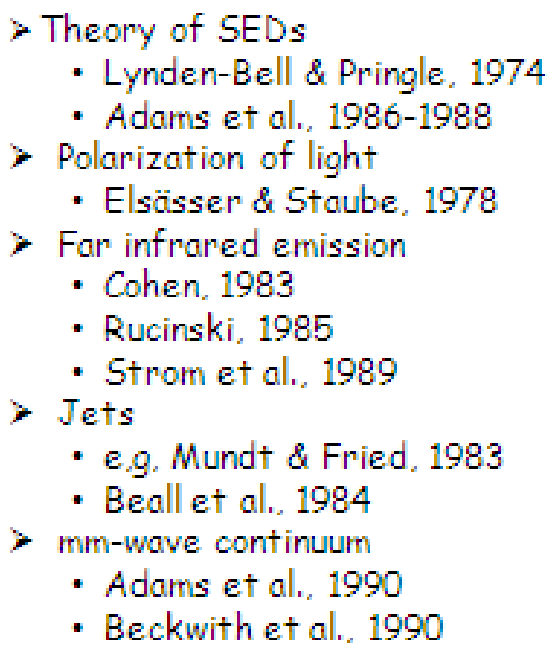

Figure 6: Inferences and proofs for the existence of disks (after Beckwith, 1998).

Figure 7 shows the SED of XX Cha in which the IR excess clearly demonstrates the presence of an optically-thick, geometrically-thin circumstellar disk (Beckwith, 1998, 1999 after Adams, Lada \& Shu, 1988).

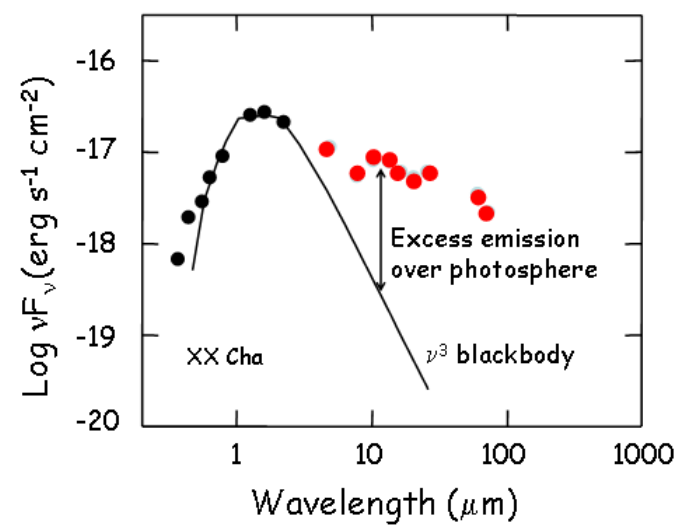

Figure 7: Spectral Energy Distribution of XX Cha (Beckwith, 1998, 1999 after Adams, Lada \& Shu, 1988).

Chiang \& Goldreich (1997) derived hydrostatic, radiative equilibrium models for passive disks surrounding T Tauri stars. Each disk is encased by an optically thin layer of superheated dust grains. This layer reemits directly to space about half the stellar energy it absorbs. The other half is emitted inward and regulates the interior temperature of the disk. The heated disk flares. As a consequence, it absorbs more stellar radiation, especially at large radii, than a flat disk would. The portion of the spectral energy distribution contributed by the disk is fairly flat throughout the thermal infrared. At fixed frequency, the contribution from the surface layer exceeds that from the interior by about a 
factor 3 and is emitted at more than an order of magnitude greater radius. Spectral features from dust grains in the superheated layer appear in emission if the disk is viewed nearly face-on.

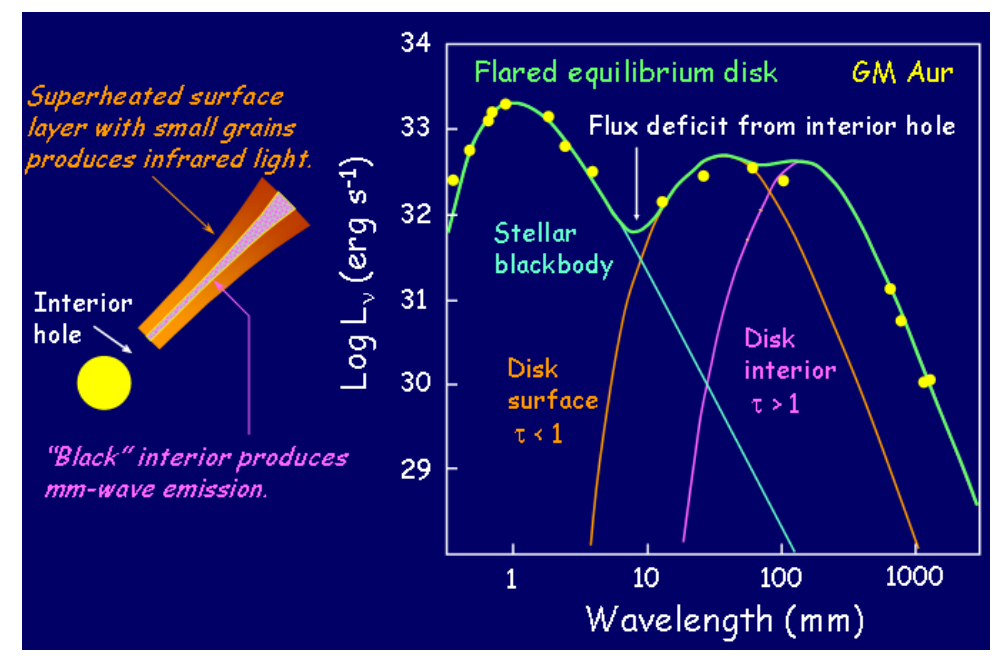

Figure 8: Observed Spectral Energy Distribution of GM Aur, and flared radiative equilibrium disk fit to GM Aur. The contribution of the different component of the system are clearly indicated (Beckwith, 1998 after Chiang \& Goldreich, 1997).

Beckwith (1998) derived from the paper by Chiang \& Goldreich (1997) the SED of the T Tauri star GM Aur, reported in Fig. 8, where the contribution of the different part of the system are clearly illustrated.

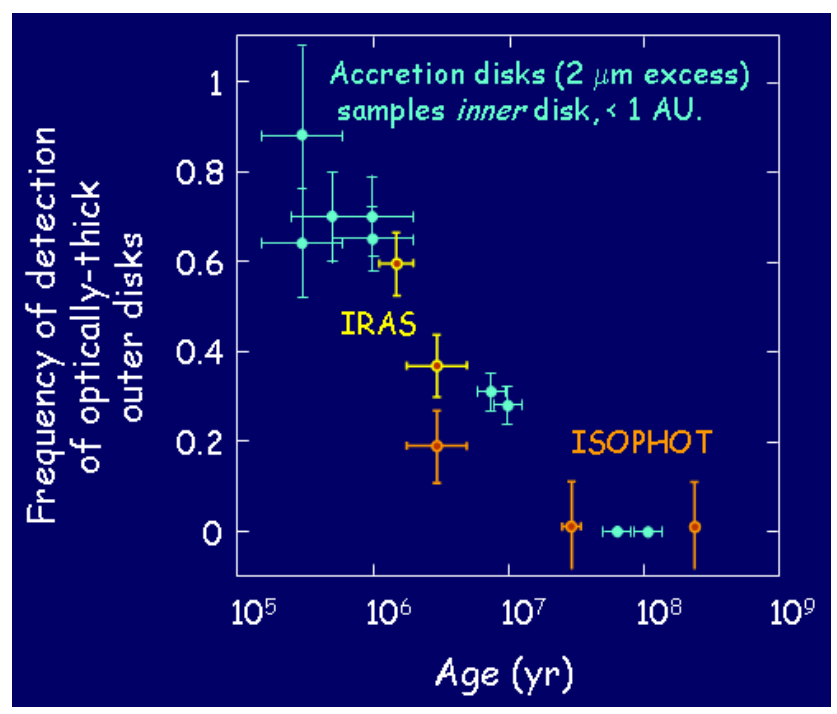

Figure 9: Frequency of detections as a function of cluster age for several star-forming regions observed by ISO, IRAS, and ISOPHOT (adapted from Beckwith, 1998 after Meyer \& Beckwith, 2000).

While it appears that most young stars form accompanied by a circumstellar disk, it is not clear that all disks form planets. Several factors could play a crucial role in determining the fate of an accretion disk. It has been suggested that disks evolve more quickly around high mass stars $\left(\mathrm{M}_{\star}>\right.$ 
$1.0 \mathrm{M}_{\odot}$ ) compared to low mass stars (Natta, Grinnin \& Mannings, 2000). Meyer \& Beckwith (2000) reviewed the observed correlations of disk properties with time as studied from ISO, IRAS, ISOPHOT. Figure 9 shows the frequency of detections of optically-thick disks as a function of cluster age for several star-forming regions observed. It appears that optically-thick disks dissipate or coagulate into larger bodies on a timescale comparable to the termination of the main accretion phase in T Tauri disks (Beckwith, 1998 after Meyer \& Beckwith, 2000).

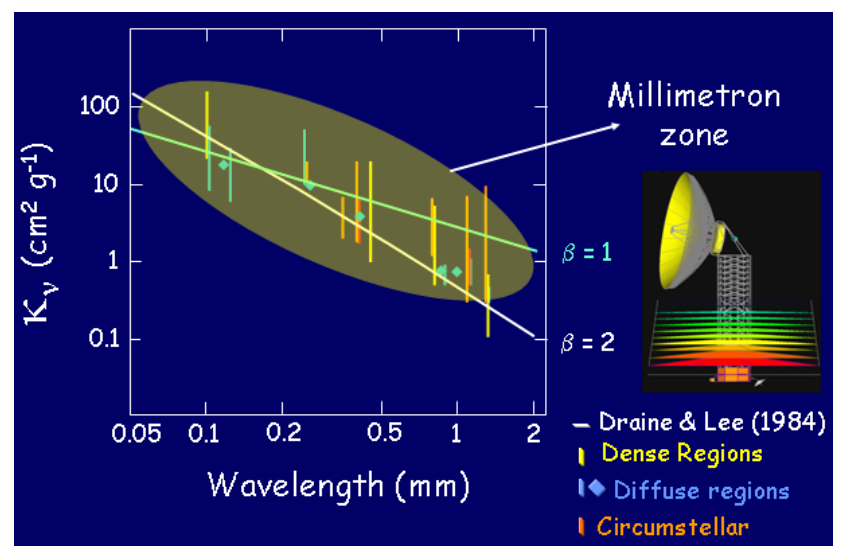

Figure 10: Uncertainties on interstellar opacities. The light brown ellipse mark the zone covered by the Millimetron (after Beckwith, 1998 and Giovannelli, 2010).

Dust opacities are relevant for dense regions in molecular clouds and for protoplanetary accretion disks. This problem was discussed by Henning, Michel \& Stognienko (1995), and the knowledge of opacities is important in order to settle the controversy among models. An important contribution will be given by the MILLIMETRON Space Observatory (Smirnov et al., 2012; Kardashev et al., 2014) which will operate in a region $(\sim 0.02-20 \mathrm{~mm})$, useful for giving answers to the uncertain values of insterstellar opacities, as shown in the Fig. 10 (light brown ellipse).

Updated Catalog of Circumstellar Disks can be found at http://www.circumstellardisks.org/ (Created by Caer McCabe. Redesigned by Isabelle H. Jansen. Maintained by Karl Stapelfeldt. Last updated April 29, 2016). The total number of resolved disks is 170, the PMS disks are 129, and the debris disks are 41.

\subsection{T Tauri Stars}

Following the paper by Linsky \& Schöller (2015) the evolution of YSOs from dense clumps of interstellar gas and dust to main sequence stars proceeds through several stages (Feigelson \& Montmerle, 1999), as shown in Fig. 3. The earliest phase of a protostar (referred to as a Class 0 object) consists of a collapsing massive disk detectable only at millimeter and far-infrared wavelengths. After $\sim 10^{5} \mathrm{yr}$, the collapsing cloud has formed a Class I object consisting of a heavily obscured star heated by the release of gravitational energy and a large circumstellar disk. After $\sim 10^{6} \mathrm{yr}$, the star becomes optically visible above and below the plane of the disk, although the visible spectrum is veiled by emission from the disk. These stars are called Classical T Tauri stars (CTTSs) or Class II objects. Strong UV emission seen in CTTSs results mostly from accretion of gas along magnetic field lines from the disk to the star, producing postshock regions with strongly 
enhanced emission in the HeI 5876 and other lines. Strong X-ray emission can be produced in the postshock region, closed-field lines in the stellar corona, and where stellar and disk magnetic fields interact. In the last stage of evolution to the main sequence, which occurs between a few million years and $\sim 10^{7} \mathrm{yr}$, most of the gas and dust from the disk has condensed into planets or has been accreted onto the star, which is now called a Weak-lined T Tauri star (WTTS) or Class III object. WTTSs are often called naked TTSs because their ultraviolet emission line spectrum is no longer obscured by a disk.

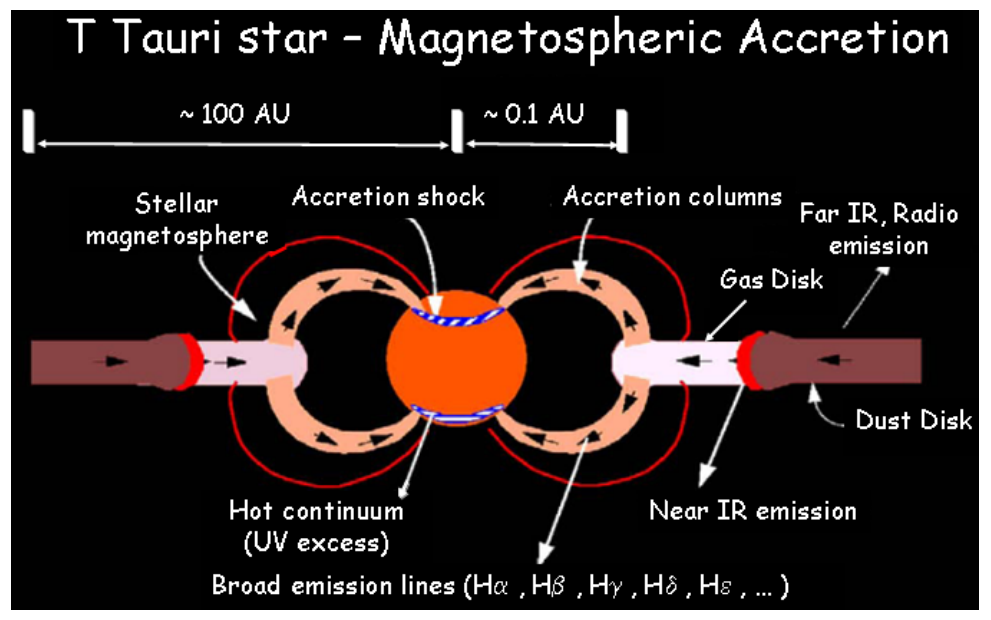

Figure 11: Magnetically funneled accretion stream producing broadened emission lines (after Hartmann, 1998 and Feigelson \& Montmerle, 1999). The dimensions marked are not in scale.

The importance of a deep study of TTSs is based among others because they probably look like the Sun in its very early stages (although with a scaling factor of $\sim 10^{3}-10^{4}$ ). TTSs are subgiant of late spectral type (later than K up to M5.5) and show high variability, strong HI, CaII, FeII emission lines, strong IR excess; the underlying photospheres are relatively cool: $\mathrm{T}<6000$ $\mathrm{K}$ (typically $\mathrm{T} \sim 4000 \mathrm{~K}$ ); masses: $0.5 \mathrm{M}_{\odot} \leq \mathrm{M} \leq 3 \mathrm{M}_{\odot}$; age: $\sim 10^{5}-10^{7} \mathrm{yr}(\mathrm{e}, \mathrm{g}$. Giovannelli, 1994).

One of the most intriguing problem is connected with the origin of magnetic field. This argument has been deeply discussed by Feigelson \& Montmerle (1999). It is difficult to study YSO magnetic fields directly, and in most cases, indirect tracers of magnetic activity such as cool star spots or high energy radiation produced by violent field reconnection must be used.

After a long multifrequency campaign of observations of the CTTS RU Lupi (Giovanelli et al., 1995) and the subsequent derived model (Lamzin et al., 1996), the proposed model by Feigelson \& Montmerle (1999, after Hartmann, 1998) - shown in Fig. 11 - can be confirmed: an accretion stream magnetically funneled produces broadened emission lines. The different zones interested in the emissions are clearly marked in Fig. 11.

\section{Formation of planets}

Beckwith et al. (1990) from a continuum observations at $1.3 \mathrm{~mm}$ of 86 pre-main-sequence stars in the Taurus-Auriga dark clouds showed that $42 \%$ have detectable emission from small particles. The detected fraction is only slightly smaller for the weak-line and "naked" T Tauri stars 
than for CTTSs, indicating that the former stars often have circumstellar material. The typical disk has an angular momentum comparable to that generally accepted for the early solar nebula, but very little stored energy, almost five orders of magnitude smaller than that of the central star. Their results demonstrate that disks more massive than the minimum mass of the proto-solar system commonly accompany the birth of solar-mass stars and suggest that planetary systems are common in the Galaxy.

This fact is supported also by the observations of submillimeter flux densities from the circumstellar disks around 29 pre-main-sequence stars (Beckwith \& Sargent, 1991). The properties of these disks are of special interest, because they bear many similarities to those of the primitive solar nebula. Studies of such systems may therefore lead to a better understanding of the formation of planetary systems like our own.

Meyer et al. (2002) discussed the formation and evolution of planetary systems utilizing the sensitivity of SIRTF (the Space Infra-Red Telescope Facility) through the Legacy Science Program to carry out spectrophotometric observations of solar-type stars. They derived circumstellar dust properties around a representative sample of primordial disks (dominated by ISM grains in the process of agglomerating into planetesimals) and debris disks (dominated by collisionally generated dust) over the full range of dust disk optical-depths diagnostic of the major phases of planet system formation and evolution. Hillenbrand (2008) discussed disk-dispersal and planet-formation timescales starting that well before the existence of exo-solar systems was confirmed, it was accepted knowledge that most - if not all - stars possess circumstellar material during the first oneto-several million years of their pre-main sequence lives, and thus that these systems commonly have the potential to form planets.

Following the very interesting paper by Machida, Inutsuka \& Matsumoto (2010) and references therein), we believe that stars are born with a circumstellar disk. The formation of the circumstellar disk is coupled with the angular momentum problem that is a serious problem in the star formation process, and the dynamics of disks may determine the mass accretion rate onto the protostar that determines the final stellar mass. In addition, planets are considered to form in the circumstellar (or protoplanetary) disk, and their formation process strongly depends on disk properties such as disk size and mass. Thus, the formation and evolution of the circumstellar disk can provide a significant clue to star and planet formation.

Stars form in molecular clouds that have an angular momentum (Arquilla \& Goldsmith, 1986; Goodman et al., 1993; Caselli, 2002; 2003). Thus, the appearance of a circumstellar disk is a natural consequence of the star formation process when the angular momentum is conserved in the collapsing cloud core. In addition, observations have shown the existence of circumstellar disks around the protostar (e.g., Watson et al., 2007; Dutrey, Guilloteau \& Ho, 2007; Meyer et al., 2007). Numerous observations indicate that circumstellar disks around Class I and II protostars have a size of $\sim 10-1000 \mathrm{AU}$ and a mass of $\sim 10^{-3}-0.1 \mathrm{M}_{\odot}$ (e.g., Calvet, Hartmann \& Strom, 2000; Natta, Grinin \& Mannings, 2000). However, they correspond to phases long after their formation. Because the formation site of the circumstellar disk and protostar are embedded in a dense infalling envelope, we cannot directly observe newborn or very young circumstellar disks (and protostars). Thus, we only observe the circumstellar disks long after their formation, i.e., around the class I or II protostar phase.

Observations also indicate that a younger protostar has a massive circumstellar disk (Natta, 
Grinin \& Mannings, 2000; Meyer et al., 2007). Enoch et al. (2009) observed a massive disk with $\mathrm{M}_{\text {disk }} \sim 1 \mathrm{M}_{\odot}$ around class 0 sources, indicating that this massive disk can be present early in the main accretion phase. However, unfortunately, observation cannot determine the real sizes of circumstellar disks, and how and when they form. Therefore, we cannot understand the formation process of the circumstellar disk by observations. The theoretical approach and numerical simulation are necessary to investigate the formation and evolution of the circumstellar disk.

For this reason Machida, Inutsuka \& Matsumoto (2010) investigated the formation and evolution of the circumstellar disk in unmagnetized molecular clouds using three-dimensional hydrodynamic simulations from the prestellar core until the end of the main accretion phase. Their calculations indicate that the planet or brown-dwarf mass object may form in the circumstellar disk in the main accretion phase. In addition, the mass accretion rate onto the protostar shows strong time variability that is caused by the perturbation of proto-planets and/or the spiral arms in the circumstellar disk. Such variability provides a useful signature for detecting the planet-sized companion in the circumstellar disk around very young protostars.

Drouart et al. (1999) starting from the fact that each nebula disk is characterized by its initial mass $\mathrm{M}_{\mathrm{D}}$, its initial radius $\mathrm{R}_{\mathrm{D}}$, and the coefficient of turbulent viscosity $\alpha$, they show that these parameters may be constrained by comparing temperature-density profiles to properly chosen physical and chemical Solar System data. They developed an analytical model that permits fruitful comparisons with available Solar System data and helpful constraints on the structure of the primitive solar nebula, as summarized below:

- Theories of the formation of giant planets provide relatively weak constraints on the main parameters which define the nebula, namely the initial mass $M_{D}$, the initial radius $R_{D}$, and the coefficient of turbulent diffusion $\alpha$. However, they conclude that forming the cores of giant planets in a time compatible with the lifetime of the nebula requires a $\alpha$ value equal to at least a few $10^{-4}$.

- The enrichment in fossil deuterium enrichment in water with respect to the protosolar abundance is by far more constraining. At $t=0, R_{D}$ must be between 8 and $28 \mathrm{AU}, \mathrm{M}_{\mathrm{D}}$ between 0.03 and $0.3 \mathrm{M}_{\odot}$, and $\alpha$ must be between 0.003 and 1 .

- The high viscosity disks they infer $(0.02<\alpha<1)$ are characterized by a MHD turbulence while their low viscosity models $(0.003<\alpha<0.02)$ are charaterized by hydrodynamical turbulence. Other mechanisms susceptible to generating a turbulent nebula compatible with observations seem to be ruled out.

- The strength of magnetic fields calculated from their selected models of nebula is consistent with the remanent magnetism found in carbonaceous chondrites. The lifetime of magnetic fields was short, no more than $10^{5}$ years in the extreme case.

Now we can say that over the past 20 years abundant evidence has emerged that many (if not all) stars are born with circumstellar disks. Understanding the evolution of post-accretion disks can provide strong constraints on theories of planet formation and evolution.

Meyer et al. (2007) reviewed on developments in understanding: i) the evolution of the gas and dust content of circumstellar disks based on observational surveys, highlighting new results from 
the Spitzer Space Telescope; ii) the physical properties of specific systems as a means to interpret the survey results; iii) theoretical models used to explain the observations; iv) an evolutionary model of our own solar system for comparison to the observations of debris disks around other stars; and v) how these results impact our assessment of whether systems like our own are common or rare compared to the ensemble of normal stars in the disk of the Milky Way.

With a sample of $\sim 328$ stars ranging in age from $\sim 3$ Myr to $\sim 3$ Gyr, Meyer et al. (2006) traced the evolution of circumstellar gas and dust from primordial planet-building stages in young circumstellar disks through to older collisionally generated debris disks. In addition to the observational program, they coordinated a concomitant theoretical effort aimed at understanding the dynamics of circumstellar dust with and without the effects of embedded planets, dust spectral energy distributions, and atomic and molecular gas line emission. Together with the observations, these efforts can provide an astronomical context for understanding whether our solar system - and its habitable planet - is a common or a rare circumstance.

The $2^{\text {nd }}$ JEDI (JEts and Disks at INAF) meeting about Jets, disks and the dawn of planets has been very interesting. The proceedings contain papers about: i) Early Protostellar Phases; ii) Disk Accretion and YSOs; iii) Jets and Winds; iv) Physical and Chemical Properties of Disks (Antoniucci et al., 2015).

Protoplanetary disks dissipate rapidly after the central star forms, on time-scales comparable to those inferred for planet formation. In order to allow the formation of planets, disks must survive the dispersive effects of UV and X-ray photoevaporation for at least a few Myr. Viscous accretion depletes significant amounts of the mass in gas and solids, while photoevaporative flows driven by internal and external irradiation remove most of the gas. A reasonably large fraction of the mass in solids and some gas get incorporated into planets. Gorti et al. (2016) review our current understanding of disk evolution and dispersal, and discuss how these might affect planet formation. They also discuss existing observational constraints on dispersal mechanisms and future directions. In the near future it is desirable to be able to connect disk evolution to planet formation and understand the close, and perhaps causal, correspondence between timescales for planet formation and disk dispersal.

Recently, investigations about the possible correlation between the mass accretion rate onto the central young star and the mass of the surrounding protoplanetary disk - theoretically predicted - have been performed. For instance, Manara et al. (2016) have accurately and homogeneously determined the photospheric parameters, mass accretion rate, and disk mass for an essentially complete sample of young stars with disks in the Lupus clouds. They found a correlation between the mass accretion rate and the disk dust mass, with a ratio that is roughly consistent with the expected viscous timescale when assuming an interstellar medium gas-to-dust ratio. This confirms that mass accretion rates are related to the properties of the outer disk. We expect in the near future measurements in different clouds in order to better determine such a correlation.

Considering that star and planet formation are the complex outcomes of gravitational collapse and angular momentum transport mediated by protostellar and protoplanetary disks, Kratter \& Lodato (2016) deeply discuss on the role of gravitational instability in this process. They highlight open questions related to the development of a turbulent cascade in thin disks and the role of mode-mode coupling in setting the maximum angular momentum transport rate in thick disks. Gravitational instability provides efficient angular momentum transport in relatively massive pro- 
tostellar disks. Evidence of this phase observationally remains sparse but is likely to improve in the coming years with more ALMA data.

Casanova, Lazarian \& Santos-Lima (2016) consider formation of accretion disks from a realistically turbulent molecular gas using 3D MHD simulations. In particular, they analyze the effect of the fast turbulent reconnection for the removal of magnetic flux from a disk. With their numerical simulations they demonstrate how the fast reconnection enables protostellar disk formation resolving the so-called "magnetic braking catastrophe".

What we can say about the step from disk to planet? Thanks to the unprecedented sensitivity and imaging capabilities offered by the Atacama Large Millimeter Array (ALMA) we have transformed our understanding of protoplanetary disks and, hence, of planet formation. Isella (2016) discuss the main results and caveats related to the measurement of the mass of solids in protoplanetary disks based on millimeter-wave observations. Then he present a recent analysis of the ALMA observations of the HL Tau disk, which suggests that the observed circular rings might be due to the tidal interaction between Saturn mass planets and the circumstellar material. In the conclusion, Isella (2016) argues that the existing observations of protoplanetary disks suggest that planets might form very early on, perhaps at the same time as the formation of the disk itself.

\subsection{Habitable Zone in the Milky Way and Exoplanets}

The most important questions about the possible origin of life in our Universe became a real scientific question in the last couple decades when it appeared a near certainty that other planets must orbit other stars. And yet, it could not be proven, until the early 1990's. Then, radio and optical astronomers detected small changes in stellar emission which revealed the presence of first a few, and now many, planetary systems around other stars. We call these planets "exoplanets" to distinguish them from our own solar system neighbors (http://science.nasa.gov/astrophysics/focusareas/exoplanet-exploration/).

Figure 12 shows the distribution of Kepler planet candidates by size as of January 2015 (Image Credit: NASA Ames/W Stenzel). As we can see there are 808 Earth-like planets in the neighbourhood of solar system.

The research of potential habitable exoplanets has been strongly supported during last two decades. Indeed, this field of astrophysics is now probably the most exciting since the discovery of planets Earth-like could open a serious debate about the possibility of life outside of solar system.

The list of the potential habitable exoplanets updated to 23rd July 2015 (Planetary Habitable Laboratory - PHL - University of Puerto Rico at Arecibo, http://phl.upr.edu/projects/habitableexoplanets-catalog) contains 31 objects: 10 Earth-size planets and 21 super-Earth-size planets. Figure 13 shows such potential habitable exoplanets. This list is continuously updated and the number of such exoplanets is rapidly increasing.

The Exoplanet Data Explorer is an interactive table and plotter for exploring and displaying data from the Exoplanet Orbit Database. The Exoplanet Orbit Database is a carefully constructed compilation of quality, spectroscopic orbital parameters of exoplanets orbiting normal stars from the peer-reviewed literature, and updates the Catalog of nearby exoplanets. A detailed description of the Exoplanet Orbit Database and Explorers was published by Han et al. (2014). The latest list in CSV format was updated on 2015/07/06 and is available at http://exoplanets.org/csv. 


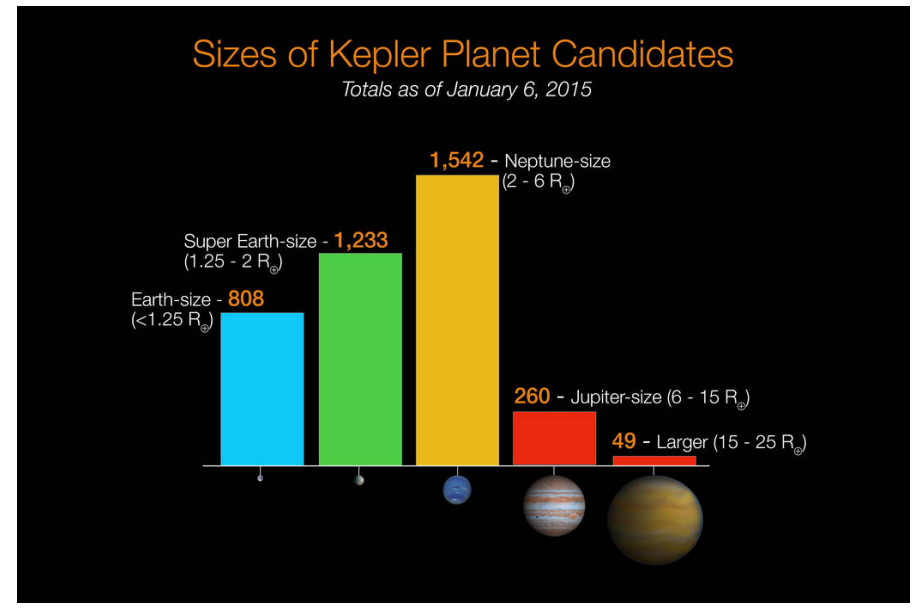

Figure 12: The distribution of Kepler planet candidates by size as of January 2015 (Image Credit: NASA Ames/W Stenzel).

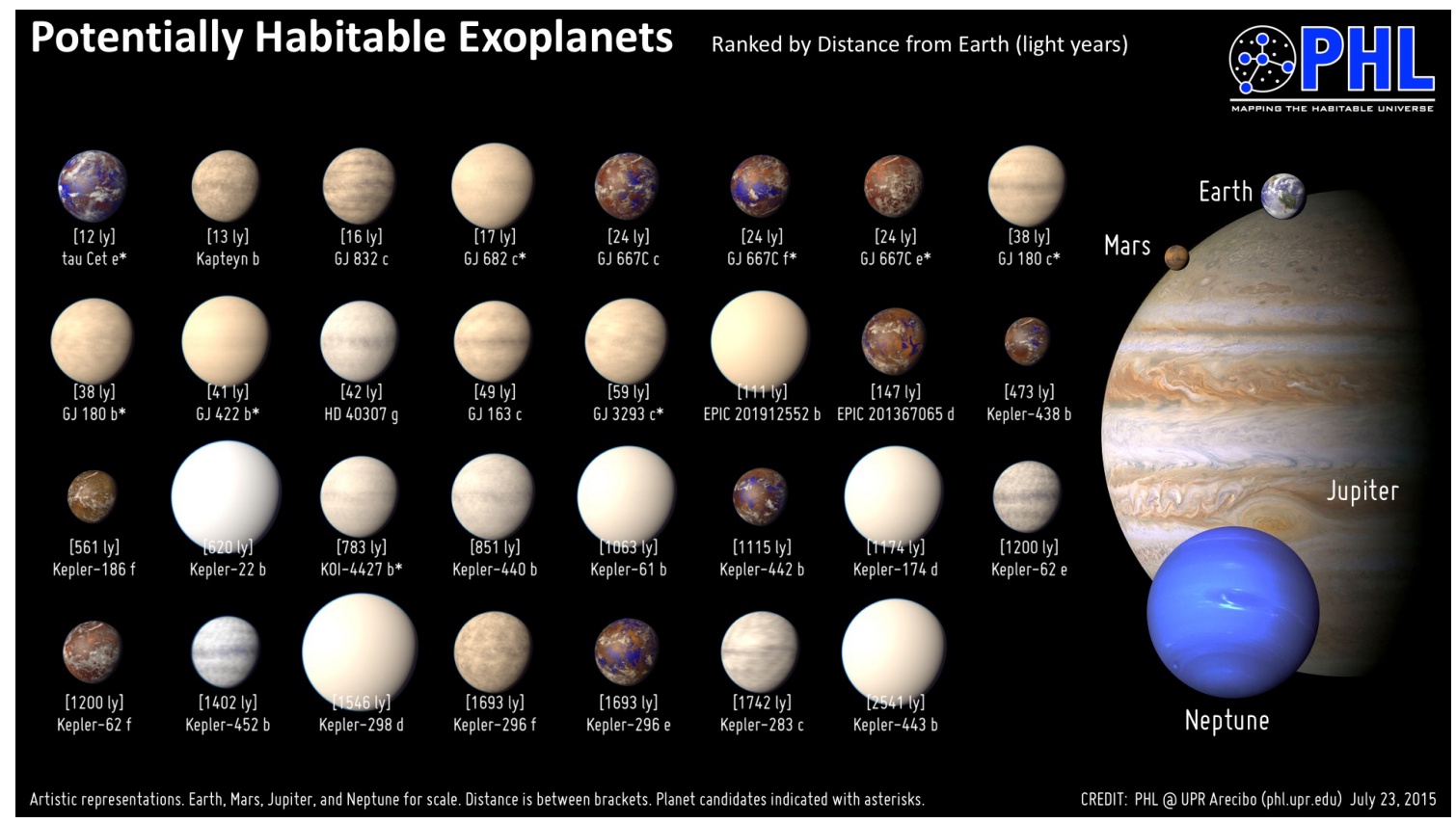

Figure 13: Current potential habitable exoplanets (2015, credit: PHL@UPR Arecibo).

In the NASA Exoplanet Archive it is possible to find updated plots about the Kepler planet candidates and all confirmed planets. Figure 14 (Nasa Exoplanet Archive) shows 4175 confirmed planets in blue (Mullally et al., 2015) among the whole set of planet candidates in red in the plane mass (Earth masses) versus period (days). It is impressive to note that there is a strong concentration of confirmed planets with masses in the range $1-10 \mathrm{M}_{\oplus}$ and periods in the range $\approx 1-100$ days.

Coughlin et al. (2016) presented the seventh Kepler planet candidate (PCs) catalog, which is the first to be based on the entire, uniformly processed, 48 month Kepler dataset. They highlight new PCs that are both potentially rocky and potentially in the habitable zone of their host stars, many of which orbit solar-type stars. This work represents significant progress in accurately 
determining the fraction of Earth-size planets in the habitable zone of Sun-like stars.

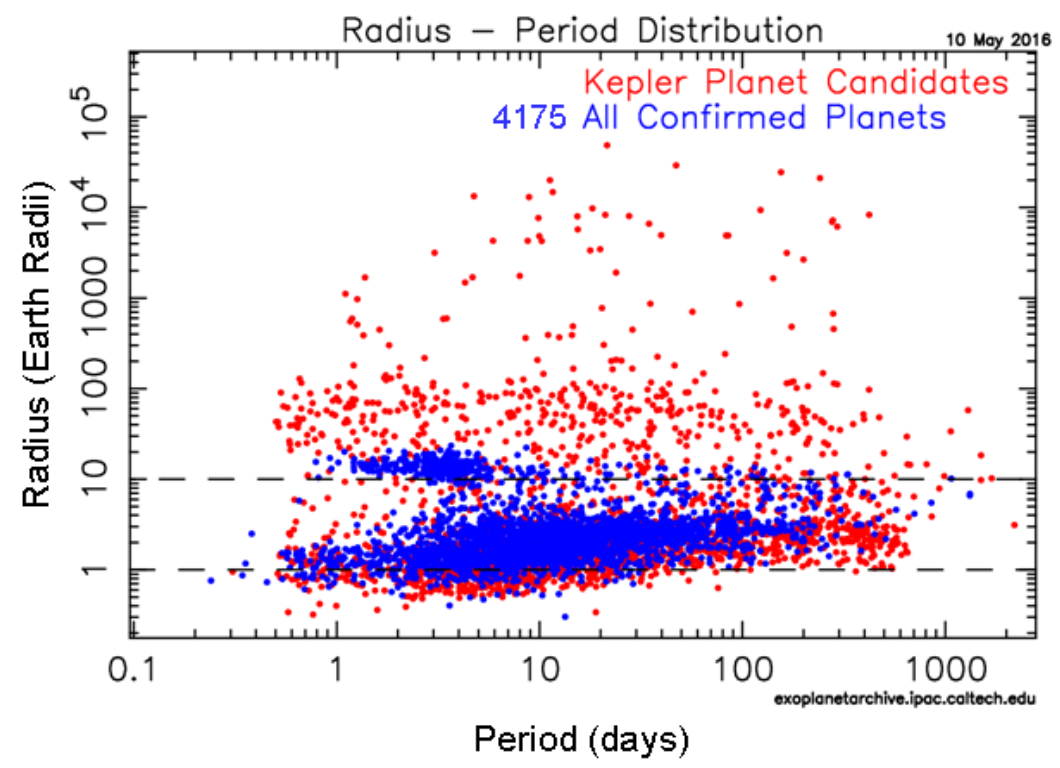

Figure 14: Radius - Period distribution of confirmed planets (blue points) and candidates planets (red points) (NASA Exoplanet Archive).

The presence of numerous exoplanets in the vicinity of solar system - within a distance of $\sim 0.8 \mathrm{pc}$ - plays an important role in speculating about the possible number of such exoplanets within the whole habitable zone of our galaxy. Such habitable zone has an internal radius of $\sim 4$ kpc and an external radius of $\sim 11 \mathrm{kpc}$, as shown in Fig. 15 (after Lineweaver, Fenner \& Gibson, 2004), where the habitable zone in a Milky Way-like galaxy is represented in green. The number of stars contained in this zone is $\approx 10 \%$ of the total number of stars in the Galaxy. Taking into account that the thickness of the disk is $\approx 1 \mathrm{kpc}$, as evaluated by the differential rotation of the Galaxy, the habitable volume is $\sim 330 \mathrm{kpc}^{3}$. Therefore, if in a volume of $\approx 2 \mathrm{pc}^{3}$ there are 808 Earth-like planets detected, in the habitable zone of our Galaxy we could expect $\approx 133 \times 10^{6}$ Earthlike planets. It is evident that the probability of finding numerous habitable planets becomes very high. Next generation instruments ground- and space-based will provide valuable information about this intriguing problem.

Studies about exoplanet predictions around stars have been performed by Bovaird \& Lineweaver (2013). They predict the existence of a low-radius $\left(R<2.5 R_{\odot}\right)$ exoplanet within the habitable zone of KOI-812 and that the average number of planets in the habitable zone of a star is 1-2.

For life-forms like us, the most important feature of Earth is its habitability. Understanding habitability and using that knowledge to locate the nearest habitable planet may be crucial for our survival as a species. During the past decade, expectations that the universe could be filled with habitable planets have been bolstered by the increasingly large overlap between terrestrial environments known to harbor life and the variety of environments on newly detected rocky exoplanets. The inhabited and uninhabited regions on Earth tell us that temperature and the presence of water are the main constraints that can be used in a habitability classification scheme for rocky planets. Lineweaver \& Chopra (2012) compiled and reviewed the recent exoplanet detections suggesting 


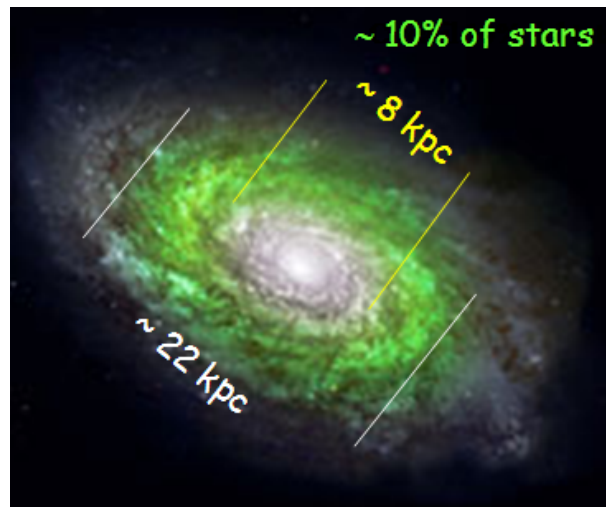

Figure 15: Habitable zone of a Milky Way-like galaxy (after Lineweaver, Fenner \& Gibson, 2004).

that the fraction of stars with planets is $\sim 100 \%$, and that the fraction with rocky planets may be comparably large. They reviewed extensions to the circumstellar habitable zone (HZ), including an abiogenesis habitable zone and the galactic habitable zone.

Earth is located in a dangerous part of the universe. Threats to life on Earth are manifold and range from asteroid impacts to supernova explosions and from supervolcano eruptions to humaninduced disasters. If the survival of the human species is to be ensured for the long term, then life on Earth has to spread to other planetary bodies. Mars is the most Earth-like planet we currently know and is the second closest planet; further it possesses a moderate surface gravity, an atmosphere, abundant water and carbon dioxide, together with a range of essential minerals. Thus, Mars is ideally suited to be a first colonization target. Here we argue that the most practical way that this can be accomplished is via a series of initial one-way human missions (Schulze-Makuch \& Davies, 2013).

However, we have interesting news about the presence of water in the universe. We knew that all the water found on Earth, has been transported by small bodies such as comets and asteroids. On the contrary, the work "The ancient heritage of water ice in the solar system" (Cleeves et al., 2014) has carried the knowledge one step further. It is understood that the water now present in Earth's oceans, and is present in other solar system bodies, has remained virtually unchanged with respect to that in the interstellar medium. This means that this water has not changed during the process of planet formation. This allows us to understand that the initial conditions that have favored the emergence of life are not unique, i.e. not dependent on the unique characteristics of our solar system. They can, however, be common in space.

Astrobiology is an interdisciplinary scientific field, recently born, not only focused on the search of extraterrestrial life, but also on deciphering the key environmental parameters that have enabled the emergence of life on Earth. Understanding these physical and chemical parameters is fundamental knowledge necessary not only for discovering life or signs of life on other planets, but also for understanding our own terrestrial environment. Recent papers by Cottin et al. (2017a,b) presented an interdisciplinary review of current research in astrobiology, covering the major advances and main outlooks in the field. They reviewed the most recent discoveries, the new understanding of planetary system formation including the specificity of the Earth among the diversity of planets, the origin of water on Earth and its unique combined properties among solvents 
for the emergence of life, the idea that the Earth could have been habitable during the Hadean Era, the inventory of endogenous and exogenous sources of organic matter and new concepts about how chemistry could evolve towards biological molecules and biological systems. In addition, many new findings show the remarkable potential life has for adaptation and survival in extreme environments. All those results from different fields of science are guiding our perspectives and strategies to look for life in other Solar System objects as well as beyond, in extrasolar worlds.

An intriguing question about the probability of finding a number of civilization in the Galaxy arises. It is now evident that Drake's formula (Drake, 1962) must be object of a robust revision.

For years, the search for manifestations of extraterrestrial civilizations has been one of humanity's most ambitious projects. Major efforts are now focused on the interception of messages from extraterrestrial civilizations, and the millimeter range is promising for these purposes (Dyson,1960). The Millimetron space observatory is aimed at conducting astronomical observations to probe a broad range of objects in the Universe in the wavelength range $20 \mu \mathrm{m}$ to $20 \mathrm{~mm}$, including the search for extraterrestrial life (Smirnov et al., 2012; Kardashev et al., 2014, and the references therein).

However, if we insist in looking for life which is like our own, why do we look for ... INTELLIGENT LIFE? (Giovannelli, 2001).

\section{Accretion onto compact objects}

For a general deep tutorial lecture see "Accretion, Black Holes, AGN and All that..." by Andrew King (http://www.exp-astro.phys.ethz.ch/seminars/LecturesunderscoreKing.pdf).

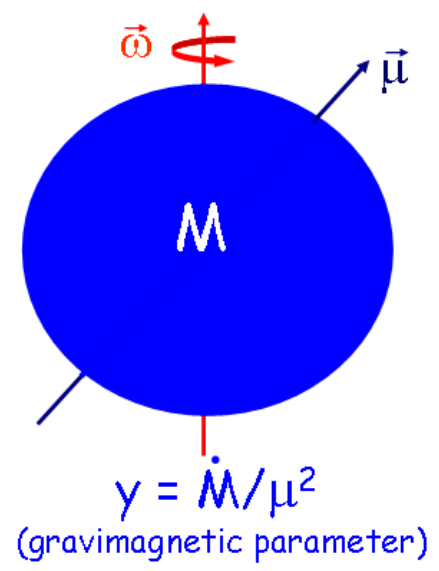

Figure 16: Gravimagnetic rotator: a body with mass $\mathrm{M}$, having a magnetic moment $\vec{\mu}$, rotating with rotational velocity $\vec{\omega}$ (Giovannelli, 2016). The parameter $\mathrm{y}=\dot{\mathrm{M}} / \mu^{2}$ is called gravimagnetic parameter (Lipunov, 1987; Lipunov \& Postnov, 1988).

Starting from the trivial definition of X-ray Binary Systems (XRBs): they are binary systems emitting X-rays, a natural question arises. Are these systems governed by few physical parameters independent of their nature? The answer is positive. Indeed, High Mass XRBs (HMXRBs), Low Mass XRBs (LMXRBs), Anomalous X-ray Pulsars (AXPs), and Cataclysmic Variables (CVs) can 
be considered as gravimagnetic rotators: a body with mass $\mathrm{M}$, having a magnetic moment $\vec{\mu}$, rotating with rotational velocity $\vec{\omega}$, being the two axis not necessarily coincident, as sketched in Fig. 16 (Giovannelli, 2016). Introducing a physical parameter, $y=\dot{M} / \mu^{2}$, named gravimagnetic parameter, all the gravimagnetic rotators are contained in a plane $\log \mathrm{P}_{\text {spin }} \mathrm{vs} \log \mathrm{y}$ (Lipunov, 1987; Lipunov \& Postnov, 1988).

The Scenario Machine (Monte Carlo simulations of binary evolution) permits to build up the complete picture of all possible evolutionary stages of binaries in the Galaxy. The basic evolution equation (5.1) used for 500,000 systems containing magnetized stars provided the results contained in the plane $\log \mathrm{P}_{\text {spin }}-\log \mathrm{y}$, reported in the upper panel of Fig. 17. $\mathrm{P}_{\text {spin }}$ is expressed in seconds and the gravimagnetic parameter is expressed in unit of $10^{-42} \mathrm{~g} \mathrm{~s}^{-1} \mathrm{G}^{-2} \mathrm{~cm}^{-6}$. The symbols used for the different types of binaries are explained in the lower panel of Fig. 17. The definition of the characteristic radii can be found in the paper by Lipunov (1987). Observational examples of various types of rotators are reported in Fig. 18 (Lipunov, 1987).

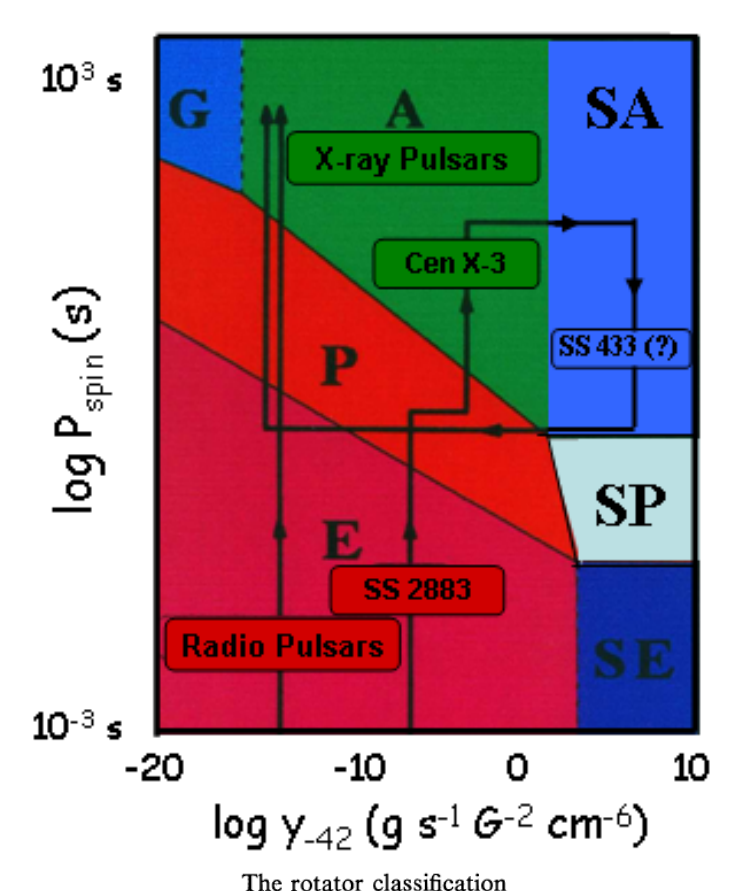

\begin{tabular}{lll}
\hline Designation & Name & Physical sense \\
\hline E & Ejector & $R_{s t}>\max \left\{R_{G}, R_{t}\right\}$ \\
P & Propeller & $R_{c}<R_{s t} \leq \max \left\{R_{G}, R_{l}\right\} \quad \dot{M}_{c} \leq \dot{M}_{c r}$ \\
A & Accretor & $R_{s t} \leq R_{G}$ and $R_{s t} \leq R_{c} \quad$ \\
G & Georotator & $R_{G}<R_{s t} \leq R_{c}$ \\
M & Magnetor & $R_{s t}>a$ and $R_{c}>a$ \\
SE & Superejector & $R_{s t}>R_{l}$ \\
SP & Superpropeller & $R_{c}<R_{s t} \leq R_{l}$ \\
SA & Superaccretor & $R_{s t} \leq R_{c}$ and $R_{s t} \leq R_{G} \quad \dot{M}_{c}>\dot{M}_{c r}$ \\
\hline
\end{tabular}

Figure 17: Upper panel: distribution of magnetic rotators in the plane "Spin Period" - "Gavimagnetic Parameter" (adapted from Lipunov, 1995); lower panel: classification of rotators (Lipunov, 1987). 


\begin{tabular}{|c|c|c|c|}
\hline Type of rotator & Designation & $\begin{array}{l}\text { The clearly confirmed } \\
\text { observational example }\end{array}$ & Model assumptions \\
\hline Ejector & $\mathrm{E}$ & Radiopulsars & $\begin{array}{l}\text { LSI }+61^{\circ} 303, \text { Cyg X-3, } \\
\text { BL Lac objects, }\end{array}$ \\
\hline Propeller & $\mathrm{P}$ & - & $\begin{array}{l}\text { Transient X-ray sources, } \\
\gamma \text {-bursts, some cataclysmic } \\
\text { variables (dwarf novae), } \\
\text { magnetic Ap-stars }\end{array}$ \\
\hline Accretor & A & $\begin{array}{l}\text { X-ray pulsars, } X \text {-ray } \\
\text { bursters, cataclysmic } \\
\text { variables with white } \\
\text { dwarfs, novae, intermediate } \\
\text { polars }\end{array}$ & - \\
\hline Superejector & SE & - & SS 433, AGN, QSO \\
\hline Superpropeller & SP & - & - \\
\hline Superaccretor & SA & - & SS 433 \\
\hline Georotator & G & - & - \\
\hline Magnetor & M & Polars & - \\
\hline
\end{tabular}

Figure 18: Observational examples of rotators (Lipunov, 1987).

$$
\frac{\mathrm{d} \mathrm{I} \omega}{\mathrm{dt}}=\dot{\mathrm{M}} K_{\mathrm{su}}-\frac{\kappa_{\mathrm{t}} \mu^{2}}{\mathrm{R}_{\mathrm{t}}^{3}}
$$

where:

$\mathrm{K}_{\mathrm{su}}=$ specific angular momentum applied by the accretion matter to the rotator;

$\mathrm{K}_{\mathrm{su}}=\sqrt{\mathrm{GM}_{\mathrm{x}} \mathrm{R}_{\mathrm{d}}}$ for Keplerian disk accretion;

$\mathrm{K}_{\mathrm{su}}=\eta_{\mathrm{t}} \Omega R_{\mathrm{g}}^{2}$ for wind accretion in a binary;

$\mathrm{K}_{\mathrm{su}} \sim 0$ for a single magnetic rotator;

$\mathrm{R}_{\mathrm{d}}=$ radius of the inner disk edge;

$\Omega=$ rotational frequency of the binary system;

$\eta_{\mathrm{t}}=1 / 4 \quad$ (Illarionov \& Sunyaev, 1975);

$\kappa_{\mathrm{t}}=$ dimensionless factor;

$\mathrm{R}_{\mathrm{t}}=$ characteristic radius;

$\dot{M}=$ accretion rate in different regimes.

Using the "Scenario Machine" Raguzova \& Lipunov (1999) obtained an evolutionary track that can lead to the formation of $\mathrm{Be} / \mathrm{BH}$ systems. The modern evolutionary scenario predicts the existence of binary black holes on eccentric orbits around Be stars and such systems may be discovered in the near future... Like happened!

Indeed, Raguzova \& Lipunov (1999) calculations show that binary black holes with Be stars must have $0.2<\mathrm{e}<0.8$. It is particularly difficult to detect such systems as most of their spectroscopic variations occur in a relatively small portion of the orbit, and could easily be missed if the systems are observed at widely separated epochs. They found for 20-30 Be/NS systems the formation of $1 \mathrm{Be} / \mathrm{BH}$ system.

Belczynski \& Ziolkowski (2009) used binary population synthesis models to show that the expected ratio of Be/XRBs with neutron stars to black holes in the Galaxy is relatively high: for $\sim$ $30-50 \mathrm{Be} / \mathrm{NS}$ systems the formation of $1 \mathrm{Be} / \mathrm{BH}$ system, and so broadly in line with observations. 


\subsection{Accretion driven X-ray sources}

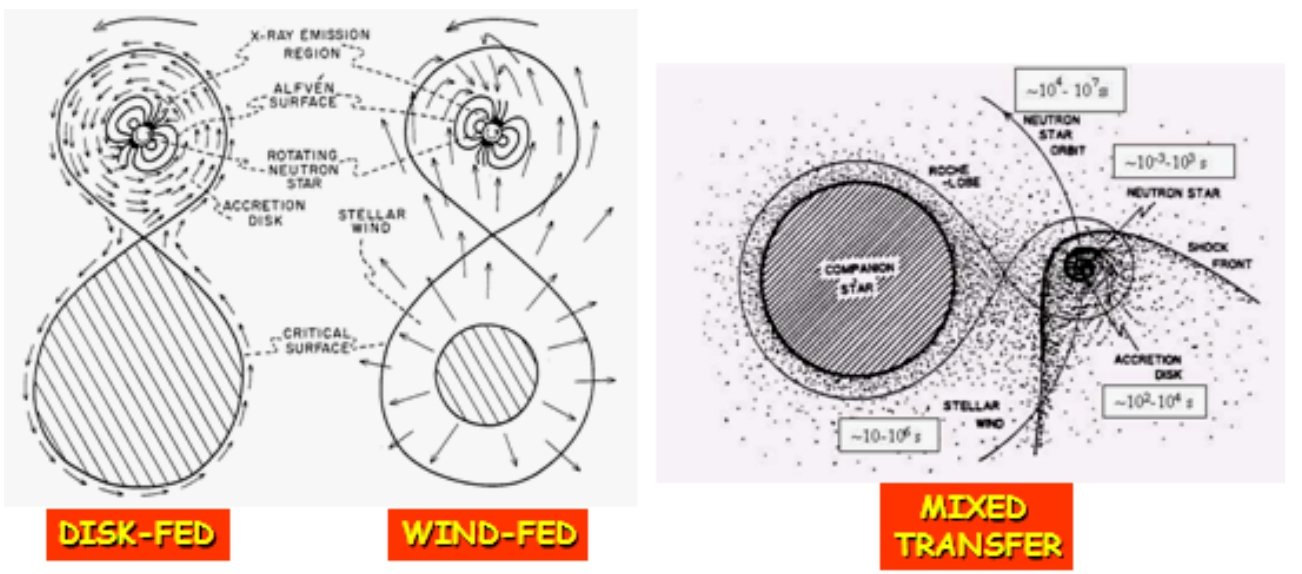

Figure 19: Left panel: accretion in X-ray binary systems disk-fed and wind-fed (Giovannelli \& SabauGraziati, 2001, adapted from Blumenthal \& Tucker, 1974). Right panel: mixed transfer (Giovannelli \& Sabau-Graziati, 2001, after Nagase, 1989).

In binary systems there are essentially two ways for accreting matter from one star to the other: via accretion disk or via stellar wind (Giovannelli \& Sabau-Graziati, 2001, adapted from Blumenthal \& Tucker, 1974) (left panel of Fig 19). But in some cases there is a third way which is a mixture between the two, as for instance in eccentric binary systems close to the periastron passage where a temporary accretion disk can be formed around the neutron star (e.g. Giovannelli \& Ziółkowski (1990), like shown in the right panel of Fig. 19 (Giovannelli \& Sabau-Graziati, 2001, after Nagase, 1989).

\subsection{Doppler tomography of accretion disks}

The fast Fourier Transform (FFT) in one, two, or three dimensions is an efficient algorithm well known for decades (see Cooley \& Tukey, 1965) as well as a traditional component of any serious numerical library. Three-dimensional (3D) FFT happens to be one of the most compute- and communication-intensive components in applications from a range of fields (for example, in turbulence, molecular dynamics, 3D tomography, and astrophysics), and for this reason there are many excellent parallel implementations in existence today. Pekurovsky (2012) introduced a popular software package called P3DFFT which implements FFT in three dimensions in a highly efficient and scalable way. This paper discussed P3DFFT implementation and performance in a way that helps guide the user in making optimal choices for parameters of their runs.

Horne (1985) and Marsh \& Horne (1988) developed Doppler tomography as a technique aimed at constructing a two-dimensional velocity image (tomogram) of the accretion disks of CVs using an emission line in its spectra sampled at a number of orbital phases. Doppler tomography has revolutionized the interpretation of orbitally phase-resolved spectroscopic observations of interacting binary systems. It has become a valuable tool for resolving the distribution of line emission in CVs and other binary systems. For instance Marsh (2001) in his review discussed the results of Doppler tomography applied to CVs. Outstanding successes to that date are the discovery of two-arm spiral 
shocks in cataclysmic variable accretion disks and the probing of the stream/magnetospheric interaction in magnetic cataclysmic variable stars. Doppler tomography has also told us much about the stream/disk interaction in non-magnetic systems and the irradiation of the secondary star in all systems.

Zhilkin \& Bisikalo (2009) described a 3D numerical code designed for simulations of magnetohydrodynamical (MHD) flows in semidetached binary systems. They found that even in case of a moderate magnetic field $\left(\mathrm{B}=10^{5} \mathrm{G}\right)$ the flow structure in the vicinity of the accretor of a $\mathrm{CV}$ was drastically changed and the funnel flows were formed in the system.

Bisikalo \& Zhilkin (2012) discussed the Flow Structure in Magnetic CVs by studying the physical processes which go on in magnetic CVs with the mass transfer between the components. Using results of 3D MHD simulations, they investigated variations of the main characteristics of accretion disks depending on the value of the magnetic induction on the surface of the accreting star. Zhilkin, Bisikalo \& Mason (2012) discussed on "Full 3D MHD calculations of accretion flow structure in magnetic cataclysmic variables with strong, complex magnetic fields".

Bisikalo et al. (2008) discussed Dopppler tomography of the CV SS Cygni in quiescence. Boneva et al. (2009) discussed the Doppler mapping in the case of SS Cygni in outburst and finally Kononov et al. (2012) used Doppler tomography for studying the pre-outburst accretion disk of SS Cygni.

Zhilkin \& Bisikalo (2011) presented a 3D numerical model to simulate plasma flows in close binary systems with accretors having strong proper magnetic fields. The model is based on an assumption that plasma dynamics is determined by a slow average flow against a background of propagating MHD waves. Equations describing the slow plasma motion are obtained as a result of the corresponding averaging procedure. The model takes into account the magnetic diffusivity which is due to the current dissipation in turbulent vortexes, magnetic buoyancy and wave MHD turbulence. They presented results of the simulations for MHD flows in a polar-like binary system. Results of the simulations show that in semi-detached binary systems with strong magnetic fields $\left(\mathrm{B}=10^{7} \mathrm{G}\right)$ the flow structure can differ sufficiently from that of systems with moderate magnetic fields $\left(B=10^{5} \mathrm{G}\right)$, Accretion disks in such magnetic systems systems $\left(B=10^{7} \mathrm{G}\right)$ are not formed and the flow is collimated into the accretion stream starting from the inner Lagrange point $\mathrm{L}_{1}$ and finishing on the magnetic poles of the accretor. This flow structure corresponds to the case of polars.

Kotze, Potter \& McBride $(2015,2016)$ explored inside-out Doppler tomography for nonmagnetic and magnetic cataclysmic variables, respectively. The code they used is available at http://www.saao.ac.za/ ejk/doptomog/main.html.

Doppler tomography has been applied also to fusion plasmas giving an image of the fastion velocity distribution function in the tokamak ASDEX Upgrade (Salewski et al., 2015). It is interesting to note that Bisikalo et al. (2016) presented a method that can be used to recover the spectrum of turbulence from observations of optically thin emission lines formed in astrophysical disks. Within this method, they analyze how line intensity fluctuations depend on the angular resolution of the instrument, used for the observations. The method allows us to restore the slope of the power spectrum of velocity turbulent pulsations and estimate the upper boundary of the turbulence scale.

Doppler tomography is very useful also for analyzing the asymmetric MHD outflows/jets from 
accreting T Tauri stars. Indeed, observations of jets from young stellar objects reveal the asymmetric outflows from some sources. Dyda et al. (2015) carried out a large set of 2.5D magnetohydrodynamic simulations for axisymmetric viscous/diffusive disk accretion to rotating magnetized stars for the purpose of assessing the conditions where the outflows are asymmetric relative to the equatorial plane. Their simulations reproduce some key features of observations of asymmetric outflows of T Tauri stars. As well known, CTTSs are variable in different time-scales. One type of variability is possibly connected with the accretion of matter through the Rayleigh-Taylor instability that occurs at the interface between an accretion disk and a stellar magnetosphere. In this regime, matter accretes in several temporarily formed accretion streams or 'tongues' which appear in random locations, and produce stochastic photometric and line variability. Kurosawa \& Romanova (2013) used the results of global three-dimensional MHD simulations of matter flows in both stable and unstable accretion regimes to calculate time-dependent hydrogen line profiles. They performed a similar modeling for a star with realistic stellar parameters (i.e. V2129 Oph), and found good agreement between the model and the time series observed line profiles (Alencar et al., 2012). This example showed that the combination of the 3D MHD and 3D radiative transfer (3D+3D) models is a useful diagnostic tool for studying magnetospheric accretion processes. Examples of the magnetospheric accretion flows in both stable and unstable regimes are shown in Fig. 20.
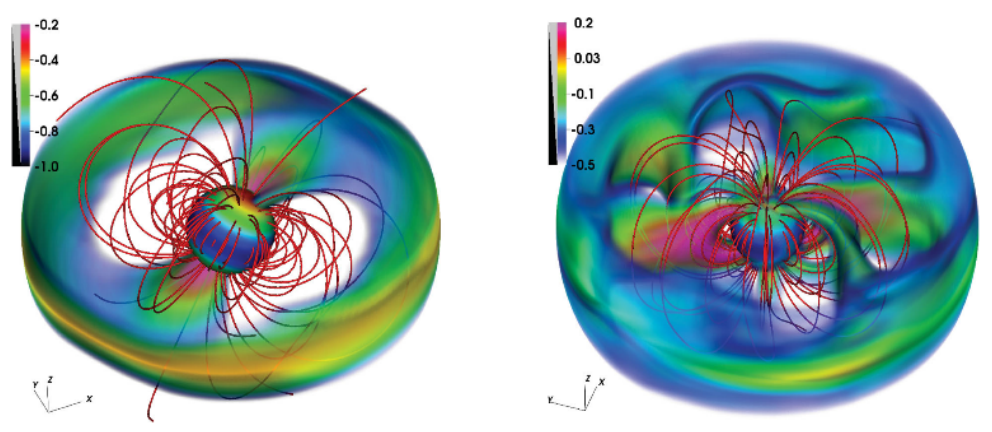

Figure 20: Magnetospheric accretions in a stable (left panel) and an unstable (right panel) regime. The background colours show the volume rendering of the density (in logarithmic scales and in arbitrary units). The sample magnetic field lines are shown as red lines (adopted from Kurosawa \& Romanova, 2013).

Lovelace \& Romanova (2014) presented results of global 3D MHD simulations of warp and density waves in accretion disks excited by a rotating star with a misaligned dipole magnetic field. Figure 21 shows a warp driven by the rotating and tilted at $\Theta=30^{\circ}$ dipole magnetosphere.

Romanova et al. (2014) reviewed axisymmetric and 3D MHD numerical simulations of magnetospheric accretion, plasma-field interaction and outflows from the disk-magnetosphere boundary. It is impressive to look at the results discussed in that paper.

Finally, we would like to remark the importance of the Doppler tomography even in planetary science. Indeed, for instance, Bisikalo, Kaygorodov \& Ionov (2013) investigated on the flow structure in systems consisting of a star and orbiting exoplanet. This is one of the most interesting problems in modern astronomy. In particular they presented the results of the numerical simulations of gas dynamics in the WASP-12 system, which was observed by the Hubble Space Telescope. The 


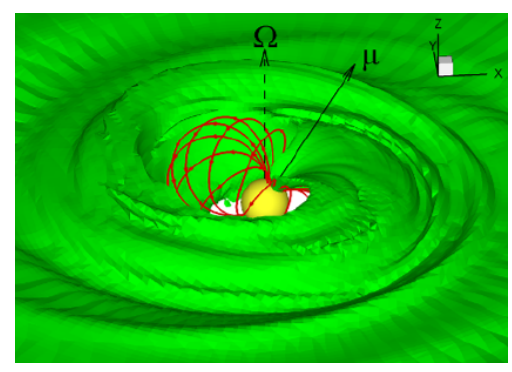

Figure 21: Example of a warp driven by the rotating and tilted at $\Theta=30^{\circ}$ dipole magnetosphere (adopted from Lovelace \& Romanova, 2014).

supersonic motion of the planet inside the stellar wind leads to the formation of a bow shock with a complex shape. The existence of the bow shock slows down the outflow through the $\mathrm{L}_{1}$ and $\mathrm{L}_{2}$ points, allowing us to consider a long-living flow structure that is in the steady state. The general morphology of the flow is shown in Fig. 22, indicating the density distribution and velocity vectors in the planet's envelope. The planet is depicted by the filled circle and moves counterclockwise. The solid yellow lines denote the flow lines starting from the gas of the stellar wind. The Roche lobe, depicted by the white solid line, and Lagrangian points $\mathrm{L}_{1}$ and $\mathrm{L}_{2}$ are also shown (Bisikalo et al., 2013).

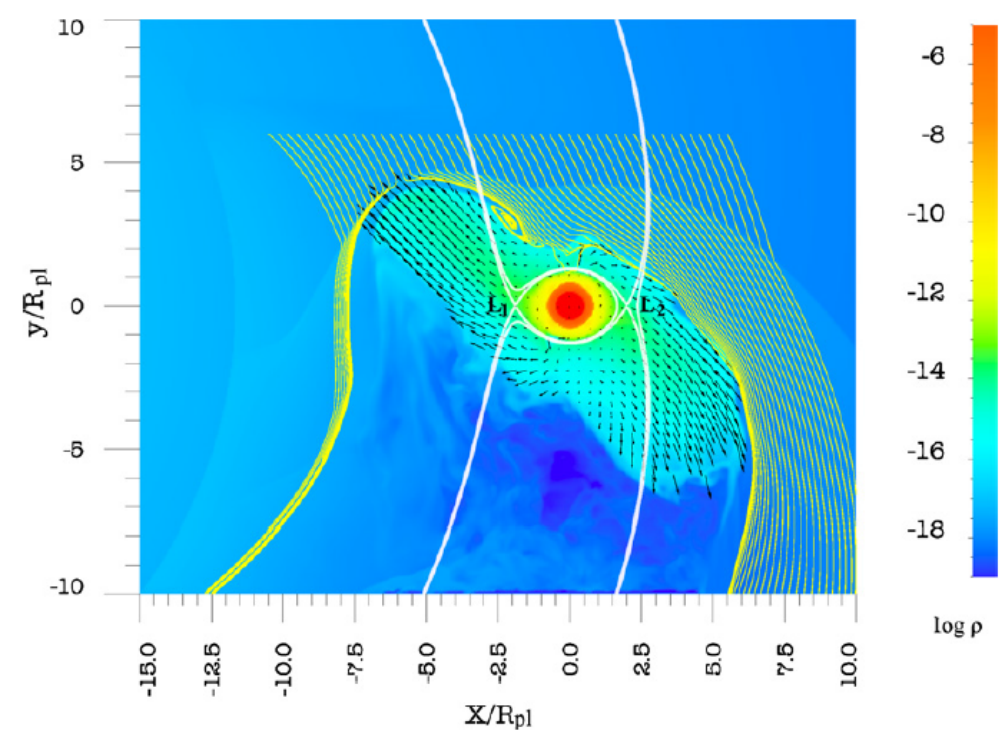

Figure 22: Density distribution and velocity vectors in the envelope of WASP-12b. The planet is depicted by the filled circle and moves counterclockwise. The solid yellow lines denote the flow lines starting in the gas of the stellar wind. The white solid lines denote the Roche equipotentials passing through the Lagrangian points $\mathrm{L}_{1}$ and $\mathrm{L}_{2}$ (adopted from Bisikalo et al., 2013).

\subsection{Accretion onto white dwarfs}

Historically, CVs - close binary systems consisting of a hot WD and red main sequence star 
of spectral type $\mathrm{M}$ or $\mathrm{K}$, which fills the volume of its inner Roche lobe and transfers matter to the vicinity of the WD - classification was based on the optical outburst properties, by which one may distinguish four groups: (i) classical novae; (ii) recurrent novae; (iii) dwarf novae; (iv) nova-like objects.

This classification, however, is neither self-consistent nor adequate and it is much better to consider primarily the observed accretion behaviour (Smak, 1985). The accretion behaviour are dependent on the WD magnetic field intensity.

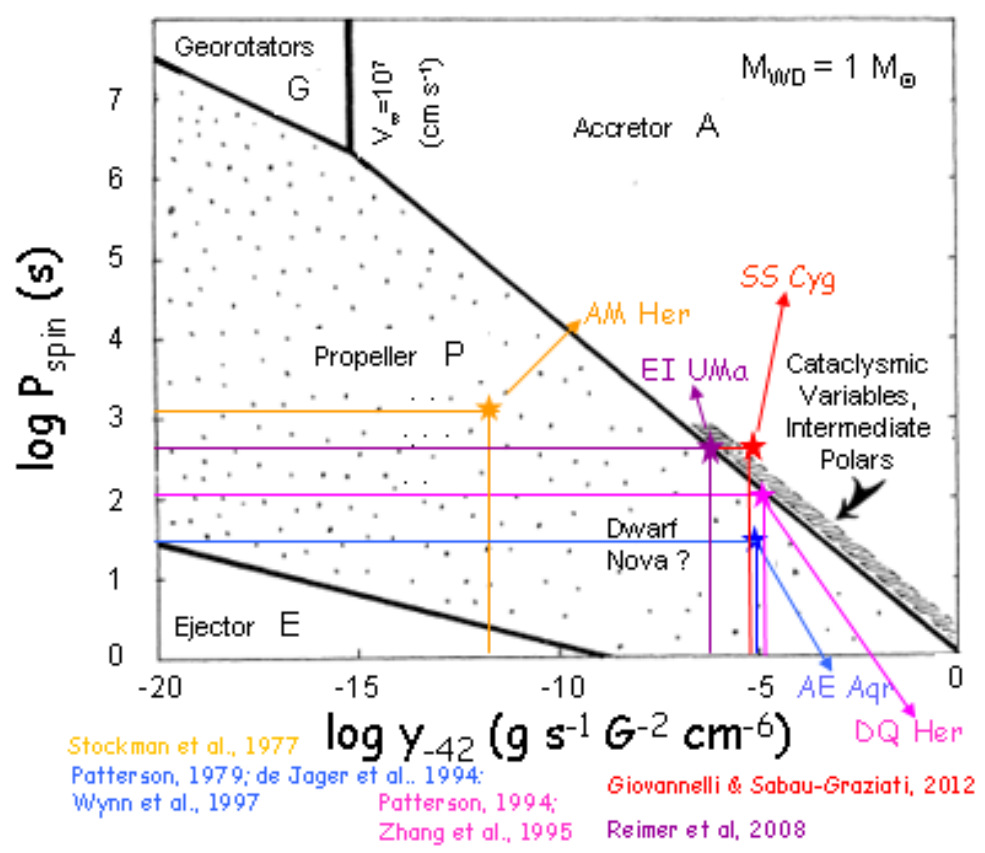

Figure 23: The position of several polars and IPs in Lipunov's diagram.

According to strength of WD magnetic field this matter is creating an accretion disk $\left(\mathrm{B} \approx 10^{5}\right.$ $\mathrm{G})$ or follows magnetic field lines and falls to surface of the $\mathrm{WD}\left(\mathrm{B} \approx 10^{6}-10^{8} \mathrm{G}\right)$. If $\mathrm{B} \approx 10^{7}-10^{8}$ $\mathrm{G}$, an accretion flow is directly channeled down towards the magnetic poles of the WD along the magnetic field lines (polars). If $\mathrm{B} \approx 10^{6}-10^{7} \mathrm{G}$, the accretion disk is not entirely disrupted, but simply truncated in its inner part, and the accretion flow is also channeled onto the WD surface along the magnetic field lines (Intermediate polars).

At the WD poles a stand-off shock is formed. The post-shock accretion column is thought to cool via bremstrahlung (hard X-rays), recombination processes and cyclotron radiation. The relative proportion of these cooling mechanisms strongly depends on the WD magnetic field (Fischer \& Beuermann 2001). An interesting discussion about Dissecting accretion and outflows in accreting white dwarf binaries is reported by de Martino et al. (2015).

Therefore, the role of magnetic field intensity plays a fundamental role in the process of accretion of matter onto the compact star. Therefore Lipunov's diagram ( $\log \mathrm{P}_{\text {spin }}$ vs $\left.\log \mathrm{y}\right)$ appears as the best way for localizing the position of MWDs, both polars and IPs, as shown in Fig. 23. 


\subsection{Accretion onto neutron stars}

$\mathrm{X}$-ray/Be systems are formed by a compact star and an optical star. Obviously there is a mutual influence between the two stars. Low-energy (LE) processes influence high-energy (HE) processes and vice versa. Never confuse the effect with the cause. There is a general law in the Universe: Cause and Effect. The Cause generates an Effect and NOT vice versa!

Time-lag between HE events and LE events in disk-fed accreting X-ray binaries (XRBs) has been noted in many systems, but the trigger of the work resulted in a model for explaining in general such a phenomenon (Bisnovatyi-Kogan \& Giovannelli, 2017) was given by Giovannelli \& SabauGraziati (2011) who noted a systematic delay between the relative enhancement in luminosity of the optical Be star - occurring at the periastron passage of the neutron star - and the subsequent X-ray flare in the system HDE 245770/A 0535+26. The model for such a system was developed and corroborated by many events (Giovannelli, Bisnovatyi-Kogan \& Klepnev, 2013: GBK13), and later by events reported in Giovannelli et al. (2015) where also a relationship between $\Delta \mathrm{V}_{\text {mag }}$ of the optical star at the periastron and X-ray intensity $\left(\mathrm{I}_{\mathrm{X}}\right)$ of the 8-day delayed flare was produced.

Briefly, the model based on an accretion disk geometrically thin and optically thick without advection (Shakura \& Sunyaev, 1973; Bisnovatyi-Kogan, 2002) is the following: in the vicinity of periastron the mass flux $\dot{M}$ increases (depending on the activity of the Be star) between $\approx 10^{-8}$ and $\approx 10^{-7} \mathrm{M}_{\odot} \mathrm{yr}^{-1}$. The outer part of the accretion disk becomes hotter, therefore the optical luminosity $\left(\mathrm{L}_{\mathrm{opt}}\right)$ increases. Due to large turbulent viscosity, the wave of the large mass flux is propagating toward the neutron star, thus the $\mathrm{X}$-ray luminosity $\left(\mathrm{L}_{\mathrm{x}}\right)$ increases due to the appearance of a hot accretion disk region and due the accretion flow channeled by the magnetic field lines onto magnetic poles of the neutron star. The time-delay $\tau$ is the time between the optical and X-ray flashes appearance.

It is right to remind that the mechanism proposed by GBK13 for explaining the X-ray-optical delay in A $0535+26 / \mathrm{HDE} 245770$ is based on an enhanced mass flux propagation through the viscous accretion disk. This mechanism, known as UV-optical delay (the delay of the EUV flash with respect to the optical flash) was observed and modeled for cataclysmic variables (e.g. Smak, 1984b; Lasota, 2001). Time delays have been detected also in several other X-ray transient binaries. This is the reason that urged Bisnovatyi-Kogan \& Giovannelli (2017) to generalize the aforementioned model, developed for the particular case of A $0535+26 / \mathrm{HDE} 245770$ (Flavia' star). This general model provides the formula (1) of the time delay in transient cosmic accreting sources:

$$
\tau=6.9 \frac{\mathrm{m}^{2 / 3} \dot{\mathrm{m}}^{1 / 15}}{\alpha^{4 / 5}\left(\mathrm{~T}_{4}\right)^{28 / 15}}
$$

where:

$\mathrm{m}=\mathrm{M} / \mathrm{M}_{\odot} ; \quad \dot{\mathrm{m}}=\dot{\mathrm{M}} /\left(10^{-8} \mathrm{M}_{\odot} / \mathrm{yr}\right) ; \quad \mathrm{T}_{4}=\mathrm{T}_{0} / 10^{4} \mathrm{~K} ; \quad \alpha=$ viscosity, and

$\mathrm{T}_{0}=$ maximum temperature in optics.

By using this formula it is possible to obtain an excellent agreement between the experimental and theoretical delays found in:

- X-ray/Be system A0535+26/HDE245770: $\tau_{\text {exp }} \simeq 8$ days (GBK13); $\tau_{t h} \simeq 8$ days; 
- Cataclysmic variable SS Cygni; $\tau_{\exp }=0.9-1.4$ days (Wheatley, Mauche \& Mattei, 2003); $\tau_{t h} \simeq 1.35$ days;

- Low-mass X-ray binary Aql X-1/V1333 Aql: $\tau_{\exp } \sim 3$ days (Shahbaz et al., 1998); $\tau_{\text {th }} \simeq$ 3.2days

- Black hole X-ray transient GRO J1655-40: $\tau_{\text {exp }} \sim 6$ days (Orosz et al., 1997); $\tau_{t h} \simeq 6.5$ days.

In this general formula the $\alpha$-viscosity parameter plays an important role, and usually it is hard to be determined. However, if the other parameters are known, because experimentally determined, the formula (5.2) can be used for determining $\alpha$, taking into account the experimental delay measured in a certain source.

This general model for the time-lag for disk-fed accreting XRBs is sketched in Fig. 24.

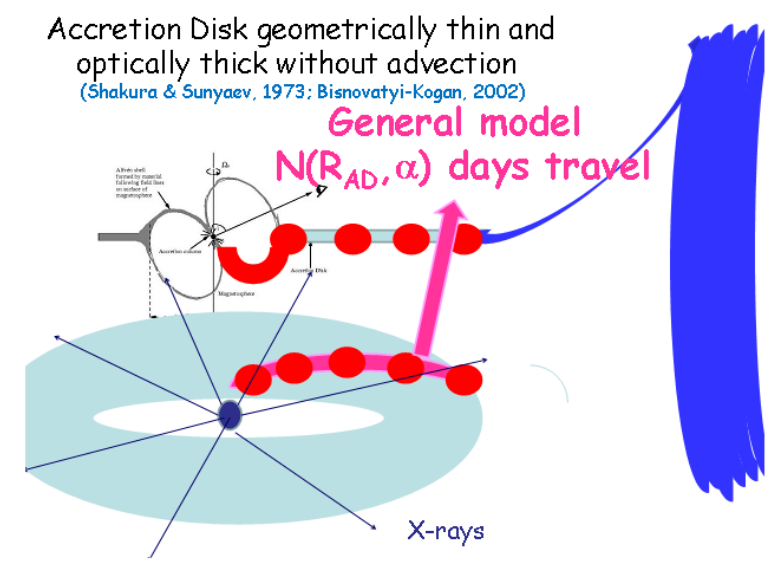

Figure 24: Time-lag general model for disk-fed accreting XRBs (Bisnovatyi-Kogan \& Giovannelli, 2017).

\subsection{Accretion onto black holes}

As already noted black hole accretion is a fundamental physical process in the universe. It is the standard model for the central engine of active galactic nuclei (AGNs), and also plays a central role in the study of black hole X-ray binaries, Gamma-ray bursts, and tidal disruption events. According to the temperature of the accretion flow, the accretion models can be divided into two classes, namely cold and hot. The standard thin disk model belongs to the cold disk, since the temperature of the gas is far below the virial value (Shakura \& Sunyaev, 1973) (see reviews by (Pringle, 1981; Frank, King \& Rayne, 2002; Abramowicz \& Fragile, 2013; Blaes, 2014). The disk is geometrically thin but optically thick and radiates multi-temperature black body spectrum. The radiative efficiency is high, $\sim 0.1$, independent of the accretion rate. The model has been successfully applied to luminous sources such as luminous AGNs and black hole X-ray binaries in the thermal state. The most recent review about the accretion onto black holes was published by Lasota (2016).

Recently several books about black holes appeared in the literature: 
- The Physics of Accretion onto Black Holes (Falanga et al., 2015). It provides a comprehensive summary on the physical models and current theory of black hole accretion, growth and mergers, in both the supermassive and stellar-mass cases.

- The Strongest Magnetic Fields in the Universe (Beskin et al. 2015). The topic of this volume contains reviews of completely new aspects of magnetic fields in the astrophysical Universe. The strength of the magnetic fields of the compact objects reviewed in this volume is up to 8 to 10 orders of magnitude higher than that of typical sunspots which are the strongest fields in the solar system. Large-scale astrophysical magnetic fields, such as interstellar and galactic fields are weaker still than the fields experienced in the solar system.

- The Formation and Disruption of Black Hole Jets (Contopoulos, Gabuzda \& Kylafis, 2015). The main aim of this book is to present reviews of the varied phenomenology regarding the radio to X-ray spectra of stellar binaries and the properties of AGN jets on the wide range of scales through which they propagate, as well as recent theoretical efforts to understand the physical mechanisms that contribute to the origin of black hole jets on all scales. Particular emphasis is given to the role and the origin of the black hole magnetosphere and the magnetic fields that drive, collimate, and accelerate the jets. The final goal is a tentative of giving a consistent, unified physical picture of the formation and disruption of jets in accreting black hole systems. New observational and theoretical results are piling up every day, so the contents of this volume only represent our current best ideas. Time will tell how close our present understanding is to reality.

- Astrophysics of Black Holes (Bambi, 2016). This book contains the most relevant papers discussed during the Fudan Winter School on Astrophysical Black Holes (held at Fudan University, in Shanghai, from February 10 to 15, 2014): Black Hole Accretion Discs, Transient Black Hole Binaries, Black Hole Spin: Theory and Observation, Winds from Black Hole Accretion Flows: Formation and Their Interaction with ISM, A Brief Review of Relativistic Gravitational Collapse, and General Relativity in a Nutshell.

- Black Holes: A Laboratory for Testing Strong Gravity (Bambi, 2017a). The main aim of this book is to discuss the electromagnetic techniques to study the strong gravity region around astrophysical black holes. For completeness, gravitational wave methods will be also reviewed, but only very briefly and without the necessary details to start working on the corresponding line of research. This book has not the ambition to be a complete manual on this research field. Hopefully, it may be a good starting point.

Important news were reported over the last couple of decades. We have witnessed the discovery of a multitude of highly ionized absorbers in high-resolution X-ray spectra from both $\mathrm{BH}$ and NS XRBs. The first detections were obtained thanks to ASCA on the BH binaries GROJ165540 and GRS 1915+105. Narrow absorption lines in the spectra of these systems identified as Fe XXV and Fe XXVI indicated the first of many discoveries of photo-ionized plasmas in LMXBs (Chandra, XMM-Newton and Suzaku).

Black hole hot accretion flows occur in the regime of relatively low accretion rates and are operating in the nuclei of most of the galaxies in the universe. One of the most important progress 
in recent years in this field is about the wind or outflow. This progress is mainly attributed to the rapid development of numerical simulations of accretion flows, combined with observations on, e.g., Sgr A*, the supermassive black hole in the Galactic center (Yuan, 2016). The mass flux of wind can be described by:

$$
\dot{\mathrm{M}}_{\text {wind }}(\mathrm{r})=\dot{\mathrm{M}}_{\mathrm{BH}}\left(\mathrm{r} / 20 \mathrm{r}_{\mathrm{s}}\right)^{\mathrm{s}}
$$

where $\mathrm{s} \approx 1$, and $\dot{\mathrm{M}}_{\mathrm{BH}}$ is the mass accretion rate at the black hole horizon and $\mathrm{r}_{\mathrm{s}}=2 \mathrm{GM} / \mathrm{c}^{2}$ is the Schwarzschild radius.

\section{Prospects for the Astrophysics of Next Decades: the Contribution of Small Experiments}

It is evident that the future of the research in astrophysics must take into account the three main methods to tackle the way of the knowledge of our Universe: photonic astrophysics, neutrino astrophysics, and particle astrophysics. The synergy of these methods converge in what we call now astroparticle physics.

With the sensitivity of the Cherenkov Telescope Array (CTA) we expect the detection of thousands VHE $\gamma$-ray sources. With the advent of the European-Extreme Large Telescope: E-ELT we will be witnesses of astonishing results. E-ELT is considered worldwide as one of the highest priorities in ground-based astronomy. It will vastly advance astrophysical knowledge in optical and near infrared (NIR) regions, allowing detailed studies of subjects including planets around other stars, the first objects in the Universe, super-massive black holes, and the nature and distribution of the dark matter and dark energy which dominate the Universe.

GAIA (e.g. Rix \& Bovy, 2013), the new generation astrometric satellite after HIPPARCOS, was launched at the end of 2013 to collect data that will allow the determination of highly accurate positions, parallaxes, and proper motions for $>10^{9}$ sources brighter than magnitude 20.7 in the white-light of its photometric band G. Gaia is providing strong impact on stellar evolution and in calibrating the energetic of cosmic sources thanks to $\sim 10 \%$ accuracy in determining the stellar distances and stellar velocities with resolution of $\sim 1 \mathrm{~km} \mathrm{~s}^{-1}$ (Prusti et al., 2016). Gaia Collaboration (Brown et al., 2016) published the "Gaia Data Release 1: Summary of the astrometric, photometric, and survey properties.

Another important jump in the knowledge of our Universe will come from the James Webb Space Telescope (JWST) that will be launched in orbit in 2018. The JWST will be a giant leap forward in our quest to understand the Universe and our origins. JWST will examine every phase of cosmic history, namely the Bridge between the Big Bang and Biology: from the first luminous glows after the Big Bang to the formation of galaxies, stars, and planets to the evolution of our own solar system. Moreover, JWST will tell us more about the atmospheres of exoplanets, and perhaps even find the building blocks of life elsewhere in the universe (see http://jwst.nasa.gov/science.html). For details about JWST see the white papers "The Scientific Capabilities of the James Webb Space Telescope" (Gardner \& the JWST Science Working Group, 2009), and numerous other white papers exploring different scientific topics at: 
https://jwst.stsci.edu/science/science-corner/white-papers.

Together with such impressive big experiments it is necessary to mention the extreme importance of small experiments that constitute useful and indispensable tools for a huge number of investigations not possible with the larger ones for a great number of reasons. They can be: i) space-based experiments: small-, mini-, micro- and nano-satellites; ii) ground-based experiments: small telescopes and robotic telescopes.

An interesting example of a micro-satellite developed by the students of Tokyo Institute of Technology is TSUBAME (launched from Russia on Nov 6, 2014) for measuring hard X-ray polarization of GRBs in order to reveal the nature of their central engine (Kurita et al., 2015). Other example about the complementarity of small mission to big experiments is TESS (The Transiting Exoplanet Survey Satellite). It will search for planets transiting bright and nearby stars. TESS is a next logical step after NASA's Kepler mission that revolutionized exoplanetary science by revealing that planets with sizes between those of the Earth and Neptune are abundant (Borucki et al., 2011; Fressin et al., 2013). TESS has been selected by NASA for launch in 2017 as an Astrophysics Explorer mission. The longest observing intervals will be for stars near the ecliptic poles, which are the optimal locations for follow-up observations with the JWST. TESS is expected to find more than a thousand planets smaller than Neptune, including dozens that are comparable in size to the Earth (Ricker et al., 2015). One example more is the study of a small satellite mission HiZ-GUNDAM for future observations of GRBs. The mission concept is to probe "the end of dark ages and the dawn of formation of astronomical objects", i.e. the physical condition of early universe beyond the redshift z $>7$ (Yonetoku et al., 2014). Small space missions, complementary to those Earth-based, even larger, are also devoted to investigate cosmic rays at very high energies, like for instance the NUCLEON space experiment designed to investigate directly, above the atmosphere, the energy spectra of cosmic-ray nuclei and the chemical composition $(Z=1-30)$ at energy range $100 \mathrm{GeV}-1000 \mathrm{TeV}$, including the "knee" energy range (Atkin et al., 2015). NUCLEON experiment on board of the RESURS-P satellite was launched on December 26, 2014.

Probably the best example of the importance of small missions is the joint JAXA/NASA ASTRO-H mission, the sixth in a series of highly successful X-ray missions developed by the Institute of Space and Astronautical Science (ISAS) (e.g. Takahashi, den Herder \& Bautz, 2014a). The launch date was decided to be on February 12, 2016. ASTRO-H is expected to provide breakthrough results in scientific areas as diverse as the large-scale structure of the Universe and its evolution, the behaviour of matter in the gravitational strong field regime, the physical conditions in sites of cosmic-ray acceleration, and the distribution of dark matter in galaxy clusters at different redshifts (Takahashi et al., 2014b - ASTRO-H Space X-ray Observatory: White Paper - in which the series of white papers, dedicated to the different topics, is listed).

As discussed by Giovannelli \& Sabau-Graziati (2016) in section 3.7 of their paper, how reionization occurred is still an open problem that deserves particular attention. Indeed, WISH (Widefield Imaging Surveyor for High-redshifts) is a space mission concept to conduct very deep and widefield surveys at NIR wavelength at $1-5 \mu \mathrm{m}$ to study the properties of galaxies at very high redshift beyond the epoch of cosmic reionization (Yamada et al., 2012).

It is important to remark the contribution to science provided by: i) Robotic Autonomous Observatories (Castro-Tirado, 2010); ii) Global Robotic Telescopes Intelligent Array for E-Science (GLORIA) (Castro-Tirado et al., 2014, 2015); iii) (B)urst (O)bserver and (O)ptical (T)ransient 
(E)xploring (S)ystem (BOOTES) (Castro-Tirado et al., 2012). With the installation of the fifth telescope of the BOOTES worldwide network of robotic telescopes it is possible a continuous monitoring of astronomical targets (Hiriart, 2014); iv) Pi of the Sky (a system of robotic telescopes designed for observations of short timescale astrophysical phenomena, e.g. prompt optical GRB emissions) (Siudek et al., 2011). PI of the Sky is now part of the GLORIA system (Mankiewicz et al., 2014; Obara et al., 2014); v) MITSuME(Multicolor Imaging Telescope for Survey and Monstrous Explosions). MITSuME telescopes were designed to perform "real time" and "automatic" follow-up observations for gamma-ray bursts (GRBs) prompted by the GCN alerts via the internet (Shimokawabe et al., 2008). An example of follow-up observations for GRBs and black hole binary is briefly reported by Saito et al. (2012, 2014); vi) CHASE (CHilean Automatic Supernova sEarch) a project began in 2007 with the goal to discover young, nearby southern supernovae in order to: i) better understand the physics of exploding stars and their progenitors; ii) refine the methods to derive extragalactic distances. During the first four years of operation, CHASE has produced more than 130 supernovae (Hamuy et al., 2012); vii) MUSICOS (MUlti-SIte COntinuous Spectroscopy) project, whose purpose is to organize multisite continuous spectroscopic observations (Baudrand \& Böhm, 1992; Foing et al., 1992); viii) WET (Whole Earth Telescope) (Nather et al., 1990). The idea born in 1986 when scientists from the University of Texas Astronomy Department established a world-wide network of cooperating astronomical observatories to obtain uninterrupted time-series measurements of variable stars. This approach has been extremely successful, and has placed the fledgling science of stellar seismology at the forefront of stellar astrophysics (e.g. Handler et al., 1996; Kilkenny et al., 2003; Reed et al., 2011); ix) MASTER (Mobile Astronomical System of the TElescope-Robots). The main goal of the MASTER-Net project is to produce a unique fast sky survey with all sky observed over a single night down to a limiting magnitude of 19-20. Such a survey will make it possible to address a number of fundamental problems: search for dark energy via the discovery and photometry of supernovae (including SNIa), search for exoplanets, microlensing effects, discovery of minor bodies in the Solar System, and space-junk monitoring. All MASTER telescopes can be guided by alerts, and realize the observations of prompt optical emission from GRBs synchronously in several filters and in several polarization planes (Lipunov et al., 2010; see also http://observ.pereplet.ru/MASTER(underscore)OT.html).

With the addition of the SAAO Telescope "farm" on the Sutherland plateau, MASTER-SAAO is giving a massive contribution to transient alerts in Astronomer's telegrams for different classes of cosmic sources, such as GRBs, SNe, Blazars, Novae, X-ray pulsars, CVs, fast rapid transient etc. (e.g at August 2015, 530 new CVs have been discovered) (Buckley, 2015).

\section{Conclusions}

After this long discussion we hope to have stressed sufficiently that the accretion processes are fundamental for explaining the physics of the vast majority of astrophysical objects. What we clearly learned is that the most critical parameter for studying the accretion processes is the $\alpha$ viscosity - very difficult to be measured -, in spite of all other parameters, such as the mass of the accretor, the diameter of the accretion disk, the size and composition of dust and grains, etc.

It appears also evident the importance of multi-frequency astrophysics from ground- and space-based experiments in order to deeply explore the nature of our Universe. 
When we discussed the prospects for the astrophysics of next decades, we hope to have given enough arguments in favor of small experiments, of course not rejecting the extremely importance of large experiments, especially those looking at the first stages of the life of our Universe. As example of small space-based experiments dedicated to specific investigations, we have mentioned TSUBAME for measuring hard X-ray polarization of GRBs, TESS for searching planets transiting bright and nearby stars, HiZ-GUNDAM for probing the end of dark ages and the dawn of formation of astronomical objects, WISH for studying the properties of galaxies at very high redshift beyond the epoch of cosmic reionization, NUCLEON for investigating directly the energy spectra of cosmic-ray nuclei and their chemical composition, and ASTRO-H, that, in spite of its small size, will provide breakthrough results in many different scientific areas. ${ }^{(*)}$

And for the ground-based small experiments we have mentioned the small telescopes (SmTs) - including those belong to amateurs - that are the unique capable of doing long-term observations of selected sources, usually forbidden with larger telescopes. SmTs - spread along the longitude and grouped in specific programs (e.g. WET, MUSICOS, BOOTES, GLORIA, MASTER) - can provide continuous long-term monitor of selected sources without night-day interruption (i.e. sdB stars for stellar seismology, RS CVn stars, XRBs, CVs, GRBs, survey of asteroids,...). Thus, SmTs - better if robotic - are unreplaceable tools complementary to larger telescopes and to ground- and space-based multi-frequency experiments.

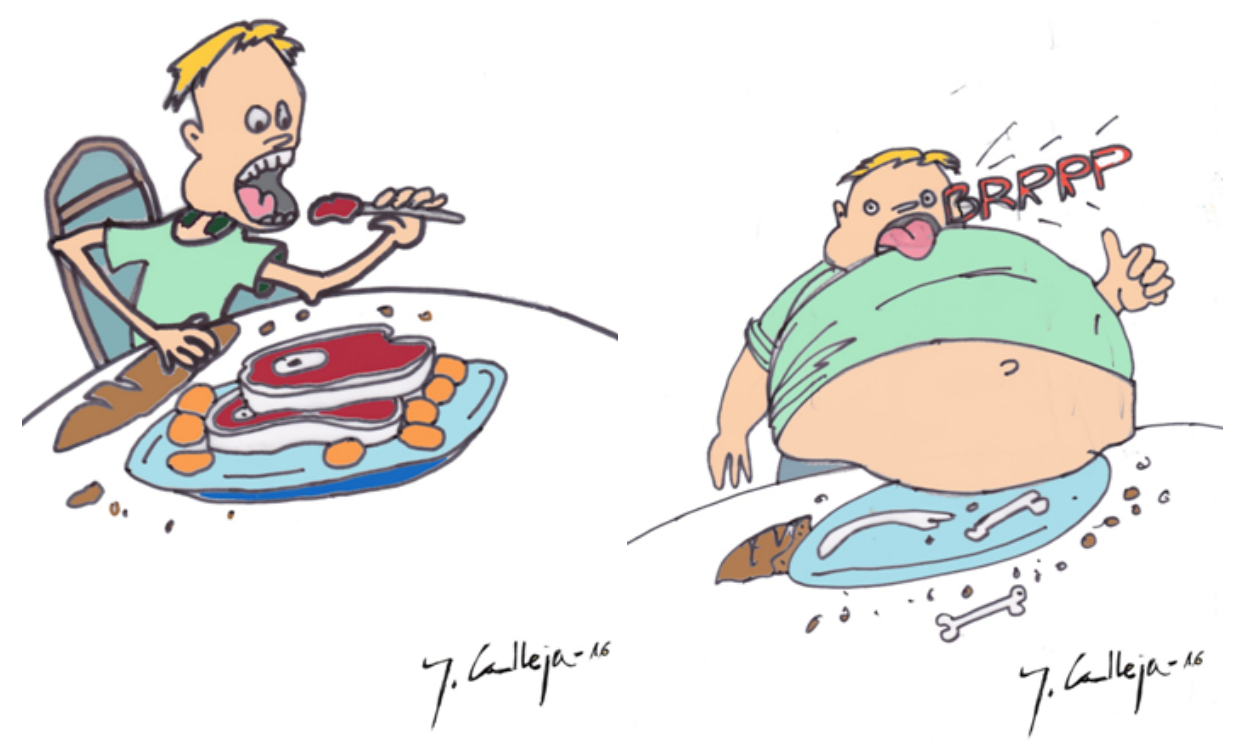

Figure 25: Accretion is a universal phenomenon (courtesy of Jaime Sanchez Calleja, 2016).

We hope also to have caused the curiosity of the reader about the investigations for discovering life or signs of life on other planets. Indeed, the Astrobiology - a recently born interdisciplinary scientific field - not only focused on the search of extraterrestrial life, but also on deciphering the key environmental parameters that have enabled the emergence of life on Earth, which is just in general sense the result of accretion processes originated in the protoplanetary disk around the Sun.

Thus, finally we can conclude that accretion is really a universal phenomenon, like shown in Fig. 25 (courtesy of Jaime Sanchez Calleja, 2016). 
${ }^{*}$ Unfortunately, during the time of the editing this review, JAXA (Japan Aerospace Exploration Agency) experienced on March 27, 2016 (JST) a communication anomaly with the of X-ray Astronomy Satellite "Hitomi" (ASTRO-H): The Japan Aerospace Exploration Agency (JAXA) found that communication with "Hitomi" (ASTRO-H) failed. The "Hitomi", launched on February 17, 2016 (JST), failed from the start of its operation, which was originally scheduled at 16:40, Saturday March 26 (JST). Up to now, JAXA has not been able to figure out the state of health of the satellite.

Acknowledgments This research has made use of:

- The NASA's Astrophysics Data System;

- the NASA Exoplanet Archive, which is operated by the California Institute of Technology, under contract with the National Aeronautics and Space Administration under the Exoplanet Exploration Program;

\section{References}

[1] Abramowicz, M.A., Fragile, P.C.: 2013, Foundations of Black Hole Accretion Disk Theory, Living Rev. Relativity 16, 1-88.

[2] Adams, F.C.: 1988, PhD Thesis, California Univ., Berkeley, USA.

[3] Adams, F.C., Shu, F.H.: 1986, ApJ 308. 836.

[4] Adams, F.C., Emerson, J.P., Fuller, G.A.: 1990, ApJ 357, 606-620.

[5] Alencar, S.H.P., Bouvier, J., Walter, F.M., Dougados, C., Donati, J.-F. et al.: 2012, A\&A 541, id. A116, 14 pp.

[6] André, P., 1994: in The Cold Universe, Montmerle Th., Lada C.J., Mirabel, I.F. \& Trân Thanh Vân, J. (Eds), Editions Frontières, Paris, p. 179.

[7] Antoniucci, S., Alcalá, J.M., Codella, C., Nisini, B. (Eds.): 2015, Disks, Jets and the dawn of planets, eprint arXiv:1506.07073.

[8] Arquilla, R., Goldsmith, P.F.: 1986, ApJ 303, 356-374.

[9] Atkin, E., Bulatov, V., Dorokhov, V., Gorbunov, N., Filippov, S. et al.: 2015, EPJ Web of Conferences, Volume 105, id.01002.

[10] Bambi, C. (Ed.): 2016, Astrophysics of Black Holes: From Fundamental Aspects to Latest Developments, Astrophysics and Space Science Library, Volume 440, 207 pp.

[11] Bambi, C.: 2017a, Black Holes: A Laboratory for Testing Strong Gravity, Springer Nature Singapore Pte Ltd., ISBN 978-981-10-4523-3, 340 pp.

[12] Bambi, C.: 2017b, Testing black hole candidates with electromagnetic radiation, PhRvD 95, Issue 10, id. 104035 .

[13] Bambi, C.: 2017c, Astrophysical Black Holes: A Compact Pedagogical Review, arXiv 171110256.

[14] Bath, G.T.: 1969, ApJ 158, 571.

[15] Bath, G.T.: 1975, MNRAS 171, 311.

[16] Bath, G.T.: 1976, in Structure and Evolution of Close Binary Systems, P. Eggleton, S. Mitton \& J. Whelan (Eds.), D. Reidel Publishing Co., IAU Symp. 73, p. 173. 
[17] Bath, G.T.: 1978, Q. Jl R. astr. Soc. 19, 442.

[18] Bath, G.T.: 1980, in Close binary stars: Observations and interpretation, Dordrecht, D. Reidel Publishing Co., IAU Symp. 88, 155-160.

[19] Bath, G.T.: 1984a, Physica Scripta T7, 101-107.

[20] Bath, G.T.: 1984b, Ap\&SS 99. 127-137.

[21] Bath, G.T., Evans, W.D., Papaloizou, J., Pringle, J.E.: 1974, MNRAS 169, 447.

[22] Baudrand, J., Böhm, T.: 1992, A\&A, 259, 711.

[23] Beall, J.H., Knight, F.K., Smith, H.A., Wood, K.S., Lebofsky, M., Rieke, G.: 1984, ApJ 284, 745-750.

[24] Beckwith, S.V.W.: 1998, talk at the The Physics of Star Formation \& Early Stellar Evolution, NATO/ASI, Crete II, 3 June.

[25] Beckwith, S.V.W.: 1999, in The Origin of Stars and Planetary Systems, Charles J. Lada \& Nikolaos D. Kylafis (Eds.), Kluwer Academic Publishers, p. 579.

[26] Beckwith, S.V.W., Sargent, A.I., Chini, R.S., Güsten, R.: 1990, AJ 99, 924-945.

[27] Beckwith, S.V.W., Sargent, A.I.: 1991, ApJ 381, 250-258.

[28] Beskin, V.S., Balogh, A., Falanga, M., Lyutikov, M., Mereghetti, S., Piran, T., Treumann, R.A. (Eds.): 2015, The Strongest Magnetic Fields in the Universe, Space Sciences Series of ISSI, Vol. 54, Springer Science+Business Media New York, ISBN 978-1-4939-3549-9, 579 pp.

[29] Birnstiel, T.: 2010, The Evolution of Gas and Dust in Protoplanetary Accretion Disks, PhD Thesis, the Ruperto-Carola University of Heidelberg, Germany. arXiv:1107.3466v1 [astro-ph.EP] 18 Jul 2011.

[30] Bisikalo, D.V., Kononov, D.A., Kaigorodov, P.V., Zhilkin, A.G., Boyarchuk, A.A.: 2008, ARep 52, 318-326.

[31] Bisikalo, D.V., Zhilkin, A.G.: 2012, in From Interacting Binaries to Exoplanets: Essential Modeling Tools, M.T. Richards \& I. Hubeny (Eds.), IAU Symp. 282, 509-516.

[32] Bisikalo, D.V., Kaygorodov, P.V., Ionov, D.E.: 2013, in Numerical Modeling of Space Plasma Flows (ASTRONUM2012), N.V. Pogorelov, E. Audit \& G.P. Zank (Eds.), ASP Conf. Ser. 474, 41-46.

[33] Bisikalo, D., Kaygorodov, P., Ionov, D., Shematovich, V., Lammer, H., Fossati, L.: 2013, ApJ 764, article id. 19, 5 pp.

[34] Bisikalo, D.V., Kurbatov, E.P., Pavlyuchenkov, Ya.N., Zhilkin, A.G., Kaygorodov, P.V.: 2016, MNRAS 458, 3892-3903.

[35] Bisnovatyi-Kogan, G.S.: 2002, in Black Hole Astrophysics 2002, Proc. of the Sixth APCTP Winter Schoo, Pohang, Korea, pp. 187-206.

[36] Bisnovatyi-Kogan, G.S., Giovannelli, F.: 2017, A\&A 599, id. A55, 7 pp.

[37] Blumenthal, G.R., Tucker, W.H.: 1974, Ann. Rev. A\&A 12, 23-46.

[38] Boneva, D., Kaigorodov, P.V., Bisikalo, D.V., Kononov, D.A.: 2009, ARep 53, 1004-1012.

[39] Borucki, W.J., Koch, D.G., Basri, G., Batalha, N., Brown, T.M. et al.: 2011, ApJ, 736, 19.

[40] Bouvier, J., Alencar, S.H.P., Harries, T.J., Johns-Krull, C.M., Romanova, M.M.: 2007, in Protostars and Planets, 951 pp., V.B. Reipurth, D. Jewitt \& K. Keil (Eds.), University of Arizona Press, Tucson, 479-494. 
[41] Bovaird, T. \& Lineweaver, C.H.: 2013, MNRAS 435, 1126.

[42] Brown, A.G.A. et al. (Gaia Collaboration): 2016, A\&A 595, id.A2, 23 pp.

[43] Buckley, D.: 2015, talk at the Palermo Workshop on "The Golden Age of Cataclysmic Variables and Related Objects - III".

[44] Burrows, C.J., Stapelfeldt, K.R., Watson, A.M., Krist, J.E., Ballester, G.E. et al.: 1996, ApJ 473, 437-451.

[45] Calvet, N., Hartmann, L., Strom, S.E.: 2000, in Protostars and Planets IV, Mannings, V., Boss, A.P., Russell, S.S. (Eds.), (Book - Tucson: University of Arizona Press), p. 377.

[46] Caselli, P.: 2002, Planetary and Space Science 50, 1133-1144.

[47] Caselli, P.: 2003, Ap\&SS 285, 619-631.

[48] Carpenter, J.M., Bouwman, J., Mamajek, E.E., Meyer, M.R., Hillenbrand, L.A. et al.: 2009, ApJS $181,197-226$.

[49] Castro-Tirado, A.J.: 2010, Hindawi Publ. Co. Adv. in Astron., Vol. 2010, ID 570489, 8 pages.

[50] Castro-Tirado, A.J., Jelínek, M., Gorosabel, J., Kubánek, P., Cunniffe, R. et al.: 2012, in Second Workshop on Robotic Autonomous Observatories, S. Guziy, S.B. Pandey, J.C. Tello \& A.J. Castro-Tirado (Eds.), ASI Conf. Ser., 7, 313-320.

[51] Castro-Tirado, A.J., Sánchez Moreno, F.M., Pérez del Pulgar, C.J., Azócar, D., Beskin, G. et al.: 2014, in Robotic Astronomy 2013, J.C. Tello, A. Riva, D. Hiriart \& A.J. Castro-Tirado (Eds.), Rev. Mex. A\&A Conf. Ser., 45, 104-109.

[52] Castro-Tirado, A.J.: 2015, in Highlights of Spanish Astrophysics VIII, Proc. of the XI Scientific Meeting of the Spanish Astronomical Society, A.J. Cenarro et al. (eds.), p. 895.

[53] Chakrabarti, S.K.: 2004, Study of Accretion Processes on Black Holes: Fifty Years of Developments, in Frontiers in Astrophysics, S.K. Chakrabarti (Ed.), Allied Publishers, p. 145, 2002. arXiv:astro-ph/0402562v1.

[54] Chiang, E.I., Goldreich, P.: 1997, ApJ 490, 368-376.

[55] Cleeves L. Ilsedore et al.: 2014, Science, 345, 1590.

[56] Cohen, M.: 1983, ApJ 270, L69.

[57] Contopoulos, I., Gabuzda, D., Kylafis, N. (Eds.): 2015, The Formation and Disruption of Black Hole Jets, Astrophysics and Space Science Library, Volume 414. ISBN 978-3-319-10355-6. Springer International Publishing Switzerland, 273 pp.

[58] Cooley, J.W., Tukey, J.W.: 1965, An algorithm for the machine calculation of complex Fourier series, Math. Comput. 19, 297-301.

[59] Cottin, H., Kotler, J.M., Bartik, K., Cleaves, H.J., Cockell, C.S., de Vera, J.-P.P. etal.: 2017a, SSRv 209, 1-42.

[60] Cottin, H., Kotler, J.M., Billi, D., Cockell, C., Demets, R. Ehrenfreund, P. et al.: 2017b, SSRv 209, 83-181.

[61] Coughlin, J.L., Mullally, F., Thompson, S.E., Rowe, J.F., Burke, C.J. et al.: 2016, ApJS, $224,12$.

[62] Drake, F.D.: 1962, Intelligent Life in Space, New York: Macmillan, 128 pp.

[63] Drouart, A., Dubrulle, B., Gautier, D., Robert, F.: 1999, Icarus 140, 129-155. 
[64] Dullemond, C.P., Hollenbach, D., Kamp, I., D’Alessio, P.: 2007, in Protostars and Planets, V,B. Reipurth, D. Jewitt \& K. Keil (Eds.), University of Arizona Press, Tucson, 951 pp., p. 555-572.

[65] Dutrey, A., Guilloteau, S., Duvert, G., Prato, L., Simon, M. et al.: 1996, A\&A 309, 493-504.

[66] Dutrey, A., Guilloteau, S., Ho, P.: 2007, in Protostars and Planets V, B. Reipurth, D. Jewitt \& K. Keil (Eds.), 951 pp., University of Arizona Press, Tucson, p. 495-506.

[67] Dyda, S., Lovelace, R.V.E., Ustyugova, G.V., Lii, P.S., Romanova, M.M., Koldoba, A.V.: 2015, MNRAS 450, 481-493.

[68] Dyson, F.: 1960, Science, 131, 1667.

[69] Efremov, Y.N., Elmegreen, B.G.: 1998, MNRAS 299, 588-594.

[70] Elmegreen, B.G.: 2002, ApJ 577, 206-220.

[71] Elsasser, H., Staude, H.J.: 1978, A\&A 70, L3.

[72] Enoch, M.L., Corder, S., Dunham, M.M., Duchêne, G.: 2009, ApJ 707, 103-113.

[73] Falanga, M., Belloni, T., Casella, P., Gilfanov, M., Jonker, P., King, A. (Eds.): 2015, The Physics of Accretion onto Black Holes, Springer, Space Sciences Series of ISSI, Vol. 49, 483 pp.

[74] Feigelson, E.D., Montmerle, T.: 1999, High-energy processes in young stellar objects, Annu. Rev. Astron. Astrophys. 37, 363-408.

[75] Fischer A., Beuermann K.: 2001, A\&A 373, 211.

[76] Foing, B.H., Catala, C., Baudrand, J., Boehm, T. (MUSICOS Team): 1992, in Cool stars, stellar systems, and the sun, Proc. of the 7th Cambridge Workshop, ASP Conf. Ser., 26, 637-642.

[77] Frank, J., King, A., Raine, D.J.: 1985, Accretion Power in Astrophysics, Cambridge and New York, Cambridge University Press, 283 pp.

[78] Frank, J., King, A., Raine, D.J.: 1992, Accretion Power in Astrophysics - Second Edition, Cambridge University Press, ISBN 0-521-40306-5, pp. xvi + 294.

[79] Frank, J., King, A., Raine, D.J.: 2002, Accretion Power in Astrophysics - Third Edition, Cambridge, UK: Cambridge University Press, pp. 398. ISBN 0521620538.

[80] Fressin, F., Torres, G., Charbonneau, D., Bryson, S.T., Christiansen, J. et al.: 2013, ApJ, 766, article id. 81,20 pp.

[81] Gardner, J.P.: 2009, Astro2010: The Astronomy and Astrophysics Decadal Survey, Science White Papers, no. 84.

[82] Giovannelli, F.: 1994, SSRv 69, 1-138.

[83] Giovannelli, F.: 2001, in The Bridge between the Big Bang and Biology (Stars, Planetary Systems, Atmospheres, Volcanoes: Their Link to Life), F. Giovannelli (ed.), President Bureau of the CNR, Roma, Italy, p. 439.

[84] Giovannelli, F.: 2008, ChJA\&A 8, Suppl. 237-258.

[85] Giovannelli, F.: 2016, http://pos.sissa.it/cgi-bin/reader/conf.cgi?confid=275, id.31.

[86] Giovannelli, F., Ziółkowski, J.: 1990, Acta Astron. 40, 95.

[87] Giovannelli, F., Vittone, A.A., Rossi, C., Errico, L., Bisnovatyi-Kogan, G.S., Kurt, V.G., Lamzin, S.A. et al.: 1995, A\&AS 114, 341. 
[88] Giovannelli, F., Sabau-Graziati, L.: 2001, Ap\&SS, 276, 67.

[89] Giovannelli, F., Sabau-Graziati, L.: 2004, The Impact of Space Experiments on our Knowledge of the Physics of the Universe, Reprinted from Space Science Reviews, Vol. 112, Nos. 1-4, pp. 1-443 (2004), Kluwer Academic Publishers, Dordrecht (GSG2004).

[90] Giovannelli, F., Sabau-Graziati, L.: 2011, Acta Polytechnica, Vol. 51, No. 2., p. 21.

[91] Giovannelli, F., Sabau-Graziati, L.: 2012, in The Golden Age of Cataclysmic Variables and Related Objects, F. Giovannelli \& L. Sabau-Graziati (eds.), Mem. S.A.It., 83 N. 2, 698-707.

[92] Giovannelli F, Bisnovatyi-Kogan, G.S., Klepnev, A.S.: 2013, A\&A 560, A1 (GBK13).

[93] Giovannelli, F., Bisnovatyi-Kogan, G.S., Bruni, I., Corfini, G., Martinelli, F., Rossi, C.: 2015, Acta Astron. 65, 107.

[94] Giovannelli, F., Sabau-Graziati, L.: 2016, in Frontier Research in Astrophysics - II, F. Giovannelli (Ed.), https://pos.sissa.it/269/001/pdf.

[95] Giovannelli, F., Bisnovatyi-Kogan, G.S.: 2017, A\&A 599, id.A55, 7 pp.

[96] González-Casanova, D.F., Lazarian, A., Santos-Lima, R.: 2016, ApJ 819, article id. 96, 11 pp.

[97] González Martínez-País, I., Shahbaz, T., Casares Velázquez, J. (Eds.): 2014, Accretion Processes in Astrophysics, Cambridge, UK: Cambridge University Press.

[98] Goodman, J.: 1993, ApJ 406, 596-613.

[99] Gorti, U., Liseau, R., Sándor, Z., Clarke, C.: 2016, SSRv 205, 125-152.

[100] Greene, T.P.: 2001, American Scientist, 89(4), 316-325.

[101] Hamuy, M., Pignata, G., Maza, J., Clocchiatti, A., Anderson, J. et al., 2012, in Multifrequency Behaviour of High Energy Cosmic Sources, Franco Giovannelli \& Lola Sabau-Graziati (Eds.), Mem. SAIt., 83, 388-392.

[102] Han, E., Wang, S., Wright, J.T., Feng, Y.K., Zhao, M. et al.: 2014, PASP, 126, 827.

[103] Handler, G., Breger, M., Sullivan, D.J., van der Peet, A.J., Clemens, J.C. et al.: 1996, A\&A, 307, 529-538.

[104] Hartmann, L.: 1998, Accretion Processes in Star Formation, Cambridge, UK; New York: Cambridge University Press, (Cambridge astrophysics series; 32). ISBN 0521435072.

[105] Hartmann, L., Kenyon, S.J.: 1987a, ApJ 312, 243-253.

[106] Hartmann, L., Kenyon, S.J.: 1987b, ApJ 322, 393-398.

[107] Hartmann, L., Herczeg, G., Calvet, N.: 2016, Accretion onto Pre-Main-Sequence Stars, ARA\&A 54, 135-180.

[108] Henning, Th., Michel, B., Stognienko, R.: 1995, P\&SS 43, 1333-1343.

[109] Hillenbrand, L.A.: 2008, Phys. Scr. T130, id. 014024, 7 pp.

[110] Hiriart, D.: 2014, Rev. Mex. A\&A Conf. Ser., 45, 87.

[111] Horne, K.: 1985, MNRAS 213, 129-141.

[112] Illarionov, A.F., Sunyaev, R.A.: 1975, A\&A 39, 185.

[113] Isella, A.: 2016, in From Interstellar Clouds to Star-Forming Galaxies: Universal Processes?, IAU Symp. 315, 107-113. 
[114] de Jager, O.C., Meintjes, P.J., O’Donoghue, D., Robinson, E.L.: 1994, MNRAS, 267, 577-588.

[115] Kardashev, N.S., Novikov, I.D., Lukash, V.N., Pilipenko, S.V., Mikheeva, E.V. et al.: 2014, Physics-Uspekhi Vol. 57, Issue 12, article id. 1199-1228.

[116] Kilkenny, D., Reed, M.D., O’Donoghue, D., Kawaler, S.D., Mukadam, A. et al.: 2003, MNRAS, 345, 834-846.

[117] Kononov, D.A., Giovannelli, F., Bruni, I., Bisikalo, D.V.: 2012, A\&A 538, id. A94, 7 pp.

[118] Kotze, E.J., Potter, S.B., McBride, V.A.: 2015, A\&A 579, id. A77, 9 pp.

[119] Kotze, E.J., Potter, S.B., McBride, V.A.: 2016, A\&A 595, id. A47, 12 pp.

[120] Kratter, K., Lodato, G.: 2016, Annu. Rev. Astron. Astrophys. 54, 271-311.

[121] Kurita S. et al. (TSUBAME Team): 2015, arXiv:1503.01975.

[122] Kurosawa, R., Romanova, M.M.: 2014, in Magnetic Fields throughout Stellar Evolution, IAU Symp. 302, 54-63.

[123] Isella, A.: 2006, Interferometric observations of pre-main sequence disks, PhD Thesis, Milano University, Italy.

[124] Isella, A., Carpenter, J.M., Sargent, A. I: 2009, ApJ 701, 260-282.

[125] Lamzin, S.A., Bisnovatyi-Kogan, G.S., Errico, L., Giovannelli, F., Katysheva, N.A., Rossi, C., Vittone, A.A.: 1996, A\&A 306, 877.

[126] Larson, R.B.: 2003, Rep. Prog. Phys. 66, 1651-1697.

[127] Lasota, J.-P.: 2001, New Astron. Rev. 45, 449.

[128] Lasota, J.-P.: 2016, Black Hole Accretion Discs, in Astrophysics of Black Holes, Cosimo Bambi (Ed.), Astrophysics and Space Science Library, Volume 440. ISBN 978-3-662-52857-0. Springer-Verlag Berlin Heidelberg, p. 1.

[129] Lazarian, A., de Gouveia Dal Pino, E.M., Melioli, C. (Eds.): 2015, Magnetic Fields in Diffuse Media, Astrophysics and Space Science Library, Volume 407. ISBN 978-3-662-44624-9. Springer-Verlag Berlin Heidelberg, 627 pp.

[130] Lewin, W.H.G., van Paradijs, J., van den Heuvel, E.P.J. (Eds.): 1995, X-ray Binaries, Cambridge Astrophysics Series, Cambridge, MA: Cambridge University Press, pp. xii + 662. ISBN 0-521-41684-1.

[131] Lin, D.N.C., Papaloizou, J.C.B.: 1996, Theory of Accretion Disks II: Application to Observed Systems, ARA\&A 34, 703-747.

[132] Lineweaver, C.H., Fenner, Y. \& Gibson, B.K.: 2004, Nature, 303, 59.

[133] Lineweaver, C.H. \& Chopra, A.: 2012, Ann. Rev. of Earth and Planetary Sci., 40 (issue 1), 597.

[134] Linsky, J.L., Schöller, M.: 2015, SSRv 191, Issue 1-4, 27-76.

[135] Lipunov, V.M.: 1987, Ap\&SS 132, 1.

[136] Lipunov, V.M., Postnov, K.A.: 1988, Ap\&SS 145, 1.

[137] Lipunov, V., Kornilov, V., Gorbovskoy, E., Shatskij, N., Kuvshinov, D., Tyurina, N. et al.: 2010, Hindawi Publ. Co. Adv. in Astron. Vol. 2010, ID 349171, 6 pages. 
[138] Lovelace, R.V.E., Romanova, M.M.: 2014, in Physics at the Magnetospheric Boundary, E. Bozzo, P. Kretschmar, M. Audard, M. Falanga \& C. Ferrigno (Eds.), EPJ Web of Conferences 64, id. 05003.

[139] Lynden-Bell, D.: 1996, Magnetic collimation by accretion discs of quasars and stars, MNRAS 279, $389-401$.

[140] Lynden-Bell, D., Pringle, J.E.: 1974, MNRAS 168, 603-637.

[141] Machida, M.N., Inutsuka, S., Matsumoto, T.: 2010, ApJ 724, 1006-1020.

[142] Manara, C.F., Rosotti, G., Testi, L., Natta, A., Alcalá, J.M. et al.: 2016, A\&A 591, id. L3, 6 pp.

[143] Mankiewicz, L., Batsch, T., Castro-Tirado, A., Czyrkowski, H., Cwiek, A. et al.: 2014, Rev. Mex. A\&A Conf. Ser., 45, 7-11.

[144] Mantle, V.J., Bath, G.T.: 1983, MNRAS 202, 151.

[145] Marsh, T.R.: 2001, in Astrotomography, Indirect Imaging Methods in Observational Astronomy, H.M.J. Boffin, D. Steeghs \& J. Cuypers (Eds.), Lecture Notes in Physics 573, 1-27.

[146] Marsh, T.R., Horne, K.: 1988, MNRAS 235, 269-286.

[147] McCaughrean, M.J., O’dell, C.R.: 1996, AJ 111, 1977-1987.

[148] de Martino, D., Sala, G., Balman, S., Bernardini, F., Bianchini, A. et al.: 2015, arXiv150102767.

[149] Menou, K.: 2008, lecture at Nordita, Stockholm, Sweden, November.

[150] Meyer, M.R., Beckwith, S.V.W.: 2000, in ISO Surveys of a Dusty Universe, D. Lemke, M. Stickel \& K. Wilke (Eds.), Lecture Notes in Physics 548, 341.

[151] Meyer, M.R., Backman, D., Beckwith, S.V.W., Brooke, T.Y., Carpenter, J.M. et al.: 2002, in The Origin of Stars and Planets: The VLT View, J.F. Alves \& M.J. McCaughrean (Eds.), ESO ASTROPHYSICS SYMPOSIA. ISBN 3-540-43541-7. Springer-Verlag, 463-470.

[152] Meyer, M.R., Hillenbrand, L.A., Backman, D., Beckwith, S., Bouwman, J. et al.: 2006, PASP 118, 1690-1710.

[153] Meyer, M.R., Backman, D.E., Weinberger, A.J., Wyatt, M.C.: 2007, in Protostars and Planets V, B. Reipurth, D. Jewitt \& K. Keil (Eds.), 951 pp., University of Arizona Press, Tucson, p. 573-588. Also arXiv:astro-ph/0606399.

[154] Meyer-Hofmeister, E., Spruit, H.: 1997, Accretion Disks - New Aspects, Lecture Notes in Physics 487, $356 \mathrm{pp}$.

[155] Mullally, F., Coughlin, J.L., Thompson, S.E., Rowe, J., Burke, C. et al.: 2015, ApJS, 217, 31 (16pp).

[156] Mundt, R., Fried, J.W.: 1983, ApJ 274, L83-L86.

[157] Nagase, F.: 1989, PASJ 41, no. 1, 1-79.

[158] Nather, R.E., Winget, D.E., Clemens, J.C., Hansen, C.J., Hine, B.P.: 1990, ApJ, 361, 309-317.

[159] Natta, A., Grinin, V., Mannings, V.: 2000, in Protostars and Planets IV, Mannings, V., Boss, A.P., Russell, S.S. (Eds.), (Book - Tucson: University of Arizona Press), p. 559-588.

[160] Obara, L., Cwiek, A., Cwiok, M., Majcher, A., Mankiewicz, L., Zarnecki, A.F.: 2014, Rev. Mex. A\&A Conf. Ser., 45, 118-122.

[161] Orosz, J.A., Remillard, R.A., Bailyn, C.D., McClintock, J.E.: 1997, ApJL 478, L83. 
[162] Pacucci, F.: 2016, The First Black Holes in the Cosmic Dark Ages, Ph.D. Thesis (Scuola Normale Superiore - Pisa, Italy), 196 pages.

[163] Pacucci, F., Volonteri, M., Ferrara, A.: 2015, MNRAS 452, Issue 2, 1922-1933.

[164] Paczyński, B.: 1965, AcA, 15, 89.

[165] Paczyński, B.: 1977, ApJ 216, 822.

[166] Papaloizou, J.C.B., Lin, D.N.C.: 1995, Theory of Accretion Disks I: Angular Momentum Transport Processes, ARA\&A 33, 505-540.

[167] Patterson, J.: 1979, ApJ 234, 978-992.

[168] Patterson, J.: 1994, PASP, 106, 209-238.

[169] Pekurovsky, D.: 2012, Siam J. Sci. Comput. Vol. 34, No. 4, pp. C192Û́C209, Society for Industrial and Applied Mathematics.

[170] Pringle, J.E.: 1981, Accretion Discs in Astrophysics, ARA\&A 19, 137-162.

[171] Prusti, T. et al. (Gaia Collaboration): 2016, A\&A 595, id.A1, 36 pp.

[172] Raguzova, N.V., Lipunov, V.M.: 1999, A\&A 349, 505.

[173] Reed, M.D., Harms, S.L., Poindexter, S., Zhou, A.-Y., Eggen, J.R. et al.: 2011, MNRAS, 412, 371-390.

[174] Reimer, T.W., Welsh, W.F., Mukai, K., Ringwald, F.A.: 2008, ApJ, 678, 376-384.

[175] Ricker, G.R., Winn, J.N., Vanderspek, R., Latham, D.W., Bakos, G.Á et al.: 2015, Journal of Astronomical Telescopes, Instruments, and Systems, Volume 1, id. 014003.

[176] Rix, H-W., Bovy, J.: 2013, A\&A Rev. 21, article id. 61.

[177] Roddier, F.: 1999, in Working on the Fringe: Optical and IR Interferometry from Ground and Space, S.C. Unwin \& R.V. Stachnik (Eds.), ASP Conf. Ser. 194. 318.

[178] Roddier, F. (Ed.): 2004, Adaptive Optics in Astronomy, Cambridge, UK: Cambridge University Press, pp. 419. ISBN 0521612144.

[179] Romanova, M.M., Lovelace, R.V.E., Bachetti, M., Blinova, A.A., Koldoba, A.V. et al.: 2014, in Physics at the Magnetospheric Boundary, E. Bozzo, P. Kretschmar, M. Audard, M. Falanga \& C. Ferrigno (Eds.), EPJ Web of Conferences 64, id. 05001.

[180] Rucinski, S.M.: 1985, AJ 90, 2321-2330.

[181] Saito, Y., Yatsu, Y., Nakajima, H., Kawai, N., Asano, K. et al.: 2012, in Death of Massive Stars: Supernovae and Gamma-Ray Bursts, P. Roming, N. Kawai \& E. Pian (Eds.), Proc. IAU Symp. No. 279, 387-388.

[182] Saito, Y., Yatsu, Y., Yoshii, T., Usui, R., Kurita, S. et al., 2014, in Suzaku-MAXI 2014: Expanding the Frontiers of the X-ray Universe, M. Ishida, R. Petre \& K. Mitsuda (Eds.), Proc. Conf. held 19-22 February, 2014 at Ehime University, Japan, p. 210.

[183] Sakurai,Y. Inayoshi, K., Haiman, Z.: 2016, MNRAS 461, 4496-4504.

[184] Salewski, M., Geiger, B., Heidbrink, W.W., Jacobsen, A.S., S B Korsholm, S.B., et al. (the ASDEX Upgrade Team): 2015, Plasma Phys. Control. Fusion 57, 014021, 10 pp.

[185] Sargent, A.I., Beckwith, S.V.W.: 1987, ApJ 323, 294-305. 
[186] Sargent, A.I., Beckwith, S.V.W.: 1989, in Structure and Dynamics of the Interstellar Medium, G. Tenorio-Tagle, M. Moles \& J. Melnick (Eds.), IAU Coll. 120, Lecture Notes in Physics, volume 350, 215-220.

[187] Scaringi, S.: 2015, talk at the Palermo Workshop on The Golden Age of CVs and Related Objects Ü III.

[188] Schulz, N.S.: 2012, The Formation and Early Evolution of Stars: From Dust to Stars and Planets, Astronomy and Astrophysics Library. ISBN 978-3-642-23925-0. Springer-Verlag Berlin Heidelberg.

[189] Schulze-Makuch, D. \& Davies, P.: 2013, JBIS, 66, 11.

[190] Shahbaz, T., Bandyopadhyay, R.M., Charles, P.A., Wagner, R.M., Muhli, P., et al.: 1998, MNRAS $300,1035$.

[191] Shakura, N.I.: 1972, Astron. Zh. 49, 921.

[192] Shakura, N.I., Sunyaev, R.A.: 1973, A\&A 24, 337.

[193] Shapiro, S.L., Teukolsky, S.A.: 1983, Black Holes, White Dwarfs, and Neutron Stars: The Physics of Compact Objects, John Wiley \& Sons, p. 396.

[194] Shimokawabe, T., Kawai, N., Kotani, T., Yatsu, Y., Ishimura, T. et al.: 2008, AIPC, 1000, 543-546.

[195] Siudek, M., Batsch, T., Castro-Tirado, A.J., Czyrkowski, H., Cwiok, M. et al: 2011, Acta Polytechnica, 51 No. 6, 64-67.

[196] Smak, J.: 1962, AcA 12, 28.

[197] Smak, J.: 1971, AcA 21, 15.

[198] Smak, J.: 1981a, AcA 31, 25.

[199] Smak, J.: 1981b, AcA 31, 395.

[200] Smak, J.: 1984a, AcA, 34, 161.

[201] Smak, J.: 1984b, PASP, 96, 5.

[202] Smak, J.: 1985, in Galactic Accreting Sources, F. Giovannelli (ed.), SIDEREA, Roma, Italy, p. 3.

[203] Smirnov, A.V., Baryshev, A.M., Pilipenko, S.V., Myshonkova, N.V., Bulanov, V.B. et al.: 2012, SPIE 8442, article id. 84424C, 9 pp.

[204] Stockman, H.S.: 1977, ApJL 218, L57-L60.

[205] Strom, K.M., Strom, S.E., Edwards, S., Cabrit, S., Skrutskie, M.F.: 1989, AJ 97, 1451-1470.

[206] Takahashi, T., den Herder, J.-W.A., Bautz, M.: 2014a, in Space Telescopes and Instrumentation 2014: Ultraviolet to Gamma Ray, Tadayuki Takahashi et al. (eds.), Proc. SPIE, 9144, 25

[207] Takahashi, T. et al. (ASTRO-H Science Working Group): 2014b, arXiv:1412.2351v1 [astro-ph.HE] 7 Dec 2014.

[208] Unsoeld, A., Baschek, B.: 2001, The new cosmos: an introduction to astronomy and astrophysics, 5th ed. Berlin: Springer, xiv, 557 p. Translated by William D. Brewer, ISBN 3540678778.

[209] Watson, A.M., Stapelfeldt, K.R., Wood, K., Ménard, F.: 2007, in Protostars and Planets V, B. Reipurth, D. Jewitt \& K. Keil (Eds.), 951 pp., University of Arizona Press, Tucson, p. 523-538.

[210] Wheatley, P.J., Mauche, C.W., Mattei, J.A.: 2003, MNRAS 345, 49. 
[211] White, R.J., Greene, T.P., Doppmann, G.W., Covey, K.R., Hillenbrand, L.A.: 2007, in Protostars and Planets, V.B. Reipurth, D. Jewitt \& K. Keil (Eds.), 951 pp., University of Arizona Press, Tucson, p. 117-132. Also arXiv:astro-ph/0604081.

[212] Wolf, S., Malbet, F., Alexander, R., Berger, J.-P., Creech-Eakman, M. et al.: 2012, A\&ARv 20, id. $52,83 \mathrm{pp}$.

[213] Wynn, G.A., King, A.R., Horne, K.: 1997, MNRAS, 286, 436-446.

[214] Yamada, T., Iwata, I., Ando, M. Doi, M., Goto, T. et al.: 2012, in Space Telescopes and Instrumentation 2012: Optical, Infrared, and Millimeter Wave, Proc. of the SPIE, Vol. 8442, article id. 84421A, $12 \mathrm{pp}$.

[215] Yonetoku, D., Mihara, T., Sawano, T., Ikeda, H., Harayama, A. et al.: 2014, Proc. of the SPIE, Vol. 9144, id. 91442 S.

[216] Zhang, E., Robinson, E.L., Stiening, R.F., Horne, K.: 1995, ApJ, 454, 447-462.

[217] Zhilkin, A.G., Bisikalo, D.V.: 2009, in Numerical Modeling of Space Plasma Flows: ASTRONUM-2008, Nikolai V. Pogorelov, Edouard Audit, Phillip Colella \& Gary P. Zank (Eds.), ASP Conf. Ser. 406, 118-123.

[218] Zhilkin, A.G., Bisikalo, D.V.: 2011, in 5th international conference of numerical modeling of space plasma flows (astronum 2010), Nikolai V. Pogorelov, Edouard Audit \& Gary P. Zank, (Eds.), ASP Conf. Ser. 444, 91-96.

[219] Zhilkin, A.G., Bisikalo, D.V., Mason, P.A.: 2012, ARep 56, 257-274. 